TRANSACTIONS OF THE

AMERICAN MATHEMATICAL SOCIETY

Volume 357, Number 10, Pages 3905-3932

S 0002-9947(05)03946-2

Article electronically published on May 20, 2005

\title{
THE POINCARÉ METRIC AND ISOPERIMETRIC INEQUALITIES FOR HYPERBOLIC POLYGONS
}

\author{
ROGER W. BARNARD, PETROS HADJICOSTAS, AND ALEXANDER YU. SOLYNIN
}

\begin{abstract}
We prove several isoperimetric inequalities for the conformal radius (or equivalently for the Poincaré density) of polygons on the hyperbolic plane. Our results include, as limit cases, the isoperimetric inequality for the conformal radius of Euclidean $n$-gons conjectured by G. Pólya and G. Szegö in 1951 and a similar inequality for the hyperbolic $n$-gons of the maximal hyperbolic area conjectured by J. Hersch. Both conjectures have been proved in previous papers by the third author.

Our approach uses the method based on a special triangulation of polygons and weighted inequalities for the reduced modules of trilaterals developed by A. Yu. Solynin. We also employ the dissymmetrization transformation of V. N. Dubinin. As an important part of our proofs, we obtain monotonicity and convexity results for special combinations of the Euler gamma functions, which appear to have a significant interest in their own right.
\end{abstract}

\section{INTRODUCTION AND MAIN RESULTS}

Hyperbolic geometry, an important and rich field of modern mathematics, has almost a two hundred year history; see [8, 18, 27, 30]. Its standard planar model suggested by H. Poincaré in 1882 - see [27] 37] for interesting historical discussions - can be realized as the unit disk $\mathbb{U}=\{z:|z|<1\}$ in the complex plane $\mathbb{C}$ supplied with the Poincaré metric

$$
d \sigma_{\mathbb{U}}(z)=\frac{|d z|}{1-|z|^{2}}, \quad z \in \mathbb{U} .
$$

Some authors [6, 37] define $d \sigma_{\mathbb{U}}$ with an extra factor 2 in the right-hand side of (1.1) enjoying the advantage of having a metric of constant curvature -1 instead of -4 in our case. However, we prefer the form (1.1), since this leads to Euclidean geometry with a standard unit of length as $z$ approaches the origin, which simplifies many of our formulas. The hyperbolic distance $\rho\left(z_{1}, z_{2}\right)$ between any two points

Received by the editors March 11, 2003.

2000 Mathematics Subject Classification. Primary 30C75; Secondary 33B15.

Key words and phrases. Isoperimetric inequality, hyperbolic geometry, Poincaré metric, polygon, conformal radius, absolutely monotonic function, Euler gamma function.

This paper was finalized during the third author's visit to Texas Tech University, 2001-2002. This author thanks the Department of Mathematics and Statistics of this University for the wonderful atmosphere and working conditions during his stay in Lubbock. The research of the third author was supported in part by the Russian Foundation for Basic Research, grant no. 00-01-00118a.

(C)2005 American Mathematical Society Reverts to public domain 28 years from publication 
$z_{1}, z_{2} \in \mathbb{U}$ can be found as

$$
\rho\left(z_{1}, z_{2}\right)=\frac{1}{2} \log \frac{1+\left|\left(z_{1}-z_{2}\right) /\left(1-\bar{z}_{1} z_{2}\right)\right|}{1-\left|\left(z_{1}-z_{2}\right) /\left(1-\bar{z}_{1} z_{2}\right)\right|} .
$$

The group of isometries of this model consists of all Möbius mappings

$$
z \rightarrow e^{i \gamma} \frac{z-z_{0}}{1-\bar{z}_{0} z}
$$

with $\gamma \in \mathbb{R}$ and $\left|z_{0}\right|<1$. The straight lines of this geometry are circular arcs and straight line segments both orthogonal to the unit circle $\mathbb{T}=\{z:|z|=1\}$, which is considered as an ideal boundary of the hyperbolic plane.

By a hyperbolic $n$-gon with $n \geq 3$ sides we mean a simply connected Jordan domain $D_{n}$ in $\mathbb{U}$, whose boundary $\partial D_{n}$ consists of $n$ hyperbolic segments, rays, or whole hyperbolic straight lines. Thus, the considered hyperbolic $n$-gons are non-convex in general and may have ideal vertices on $\mathbb{T}$; see Figure 1 Besides the role that the hyperbolic polygons play in the hyperbolic trigonometry and geometry, they also play a significant role in the theory of Fuchsian groups, Riemann surfaces, and automorphic functions, [6, 14, 25.

If $D_{n}$ has an angle $\alpha_{k} \pi$ at its vertex $a_{k}, k=1, \ldots, n$, then the hyperbolic area of $D_{n}$, which is proportional to the defect of the polygon, can be computed by the Gauss-Bonnet formula

$$
h \text {-area }\left(D_{n}\right)=\frac{\pi}{4}\left(n-2-\sum_{k=1}^{n} \alpha_{k}\right) ;
$$

see [6, §7.15]. Equality (1.2) shows, in particular, that for a fixed $n \geq 3$, the hyperbolic area of any hyperbolic $n$-gon is bounded by $\sigma_{n}=\pi(n-2) / 4$. This is one of many remarkable features distinguishing the hyperbolic and Euclidean geometries. The maximal hyperbolic area

$$
h \text {-area }\left(D_{n}\right)=\pi(n-2) / 4
$$

occurs if and only if $\alpha_{1}=\ldots=\alpha_{n}=0$. In the latter case, all the vertices of $D_{n}$ lie on the ideal boundary $\mathbb{T}$; see Figure $1 \mathrm{~b}$, which shows the regular hexagon of the maximal hyperbolic area $\sigma_{6}$ centered at $z_{0}=1 / 2$.

For a fixed $A, 0<A \leq \sigma_{n}$, let $D_{n}(A)$ denote the regular hyperbolic $n$-gon having hyperbolic area $A$, centered at the origin with one vertex on the real axis $\mathbb{R}$; see Figure 1;. As in the Euclidean case, the regular hyperbolic $n$-gons are extremal in numerous problems of hyperbolic geometry. For instance, they maximize the hyperbolic area among all hyperbolic $n$-gons with a given hyperbolic perimeter and also among all cyclic hyperbolic $n$-gons inscribed in a hyperbolic circle of a prescribed radius. A proof of the first result is outlined in [7, while the second result can be proven by a triangulation of the cyclic hyperbolic polygon. For a study of related problems, see [23. In general, extremal problems on the hyperbolic plane have been found to be much harder than their Euclidean counterparts even for purely geometric characteristics of polygons.

Other characteristics, of a non-geometrical nature, such as eigenvalues of the Laplacian, capacities, conformal radius, harmonic measure, etc., are even less accessible by the known methods and, except for the results in [15, 33, 34], there are no known sharp inequalities of this type for hyperbolic polygons with $n \geq 3$ sides.

The metric (1.1) can be transplanted onto any hyperbolic Riemann surface. More precisely, let $\mathcal{R}$ be a Riemann surface over the complex plane having a universal 


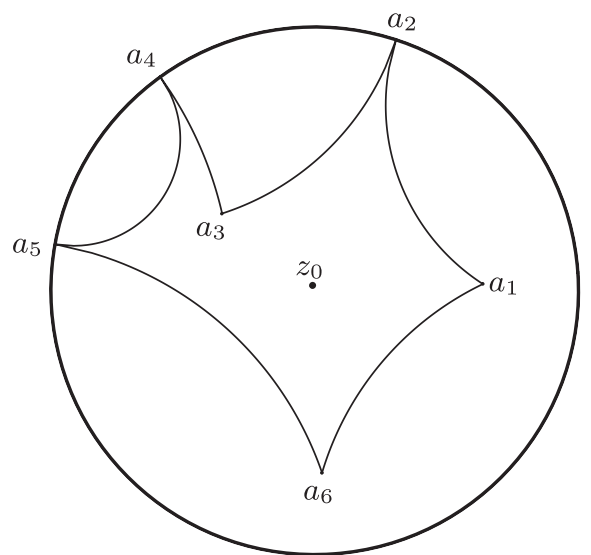

a) Non-convex 6-gon with 3 ideal vertices

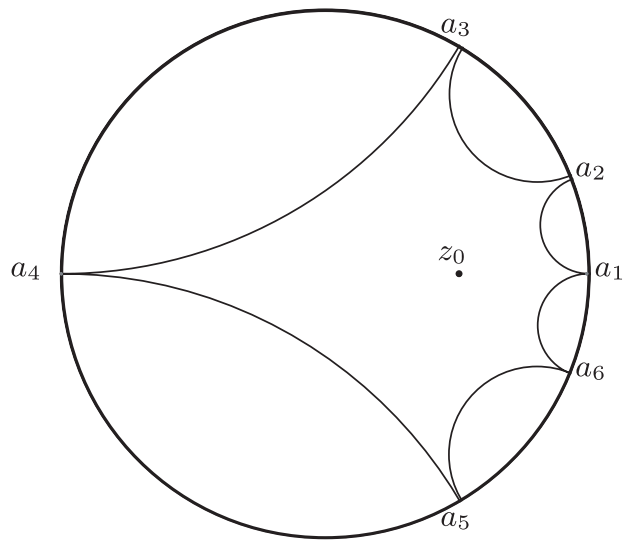

b) Regular 6-gon of the maximal h-area $\sigma_{6}$

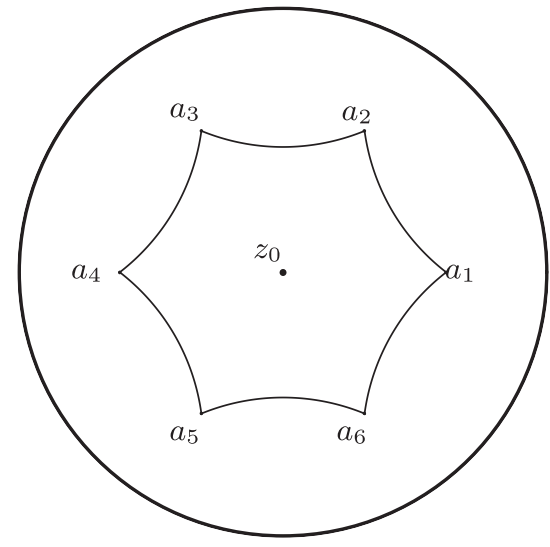

c) Regular 6-gon centered at $z_{0}=0$

FiguRE 1. Hyperbolic polygons

covering mapping $\pi_{\mathcal{R}}: \mathbb{U} \rightarrow \mathcal{R}$ (see [6, 25]), and let $p \in \mathcal{R}$. Then $\mathcal{R}$ admits a Riemannian metric of constant curvature -4 defined by

$$
d \sigma_{\mathcal{R}}(p)=\frac{|d p|}{\left|\pi_{\mathcal{R}}^{\prime}(z)\right|\left(1-|z|^{2}\right)} \quad \text { with } \quad z=\pi_{\mathcal{R}}^{-1}(p) \in \mathbb{U} .
$$

It is well known that the quantity $\left[\pi_{\mathcal{R}}^{\prime}(z)\left(1-|z|^{2}\right)\right]^{-1}$ with $z=\pi_{\mathcal{R}}^{-1}(p)$, called the Poincaré density of $\mathcal{R}$ at $p=\pi_{\mathcal{R}}(z)$, does not depend on the branch of the inverse function $\pi_{\mathcal{R}}^{-1}(p)$ and thus it is well defined. Its reciprocal $R(\mathcal{R}, p)=\left|\pi_{\mathcal{R}}^{\prime}(z)\right|\left(1-|z|^{2}\right)$ with $z=\pi_{\mathcal{R}}^{-1}(p)$ is called the conformal radius of $\mathcal{R}$ at $p$. The notion of conformal radius, usually defined for a simply connected planar domain, registers several important characteristics of $D$, among which are the geometric concept of transfinite diameter due to M. Fekete, the concept of Chebyshev's constant from polynomial approximation, and the concept of the logarithmic capacity from potential theory; see [11, $12,16,21,28,35$. 
The aim of this paper is to prove several new isoperimetric inequalities for the conformal radius (or, equivalently, for the Poincaré density) of hyperbolic $n$-gons. Since all the domains $D$ under consideration will lie on the unit disk $\mathbb{U}$, it is convenient to use a variant of the conformal radius, called the conformal h-radius of $D$ at $z_{0}, z_{0} \in D$, defined by

$$
R_{h}\left(D, z_{0}\right)=R\left(D, z_{0}\right)\left(1-\left|z_{0}\right|^{2}\right)^{-1},
$$

which is invariant under isometries of the hyperbolic plane. Note that for $z_{0}=0$, $R_{h}(D, 0)=R(D, 0)$. The maximal conformal $h$-radius $R_{h}(D)=\sup _{z \in D} R_{h}(D, z)$ is also invariant under the isometries of $\mathbb{U}$.

The principal result of this paper is the following

Theorem 1.1. Let $D_{n} \ni z_{0}$ be a hyperbolic polygon with $n \geq 3$ sides and $h$-area $A, 0<A \leq \sigma_{n}$, and let $\beta=1 / 2-1 / n-2 A / \pi n$. Then

$$
R_{h}^{2}\left(D_{n}, z_{0}\right) \leq R_{h}^{2}\left(D_{n}(A), 0\right)=\frac{\Gamma^{2}\left(1-\frac{1}{n}\right) \Gamma\left(\frac{1}{2}+\frac{1}{n}+\beta\right) \Gamma\left(\frac{1}{2}+\frac{1}{n}-\beta\right)}{\Gamma^{2}\left(1+\frac{1}{n}\right) \Gamma\left(\frac{1}{2}-\frac{1}{n}+\beta\right) \Gamma\left(\frac{1}{2}-\frac{1}{n}-\beta\right)},
$$

where $\Gamma$ denotes the Euler gamma function, with the sign of equality only for the regular hyperbolic $n$-gons centered at $z_{0}$.

In other words, Theorem 1.1 asserts that the regular hyperbolic polygon has the maximal conformal $h$-radius among all hyperbolic polygons with a fixed number of sides and prescribed hyperbolic area.

We already mentioned that extremal problems on the hyperbolic plane are much harder than their Euclidean counterparts. From this perspective, (1.4) may be the first sharp inequality for functional characteristics of $n$-gons on the hyperbolic plane known for all $n \geq 3$.

Inequality (1.4) contains as a limit case the isoperimetric inequality for the conformal radius of Euclidean polygons conjectured by Pólya and Szegö [28, p. 159].

Theorem 1.2 ([31]). Let $D_{n}$ be a Euclidean polygon having $n \geq 3$ sides. Let $R\left(D_{n}\right)=\max _{z \in D_{n}} R\left(D_{n}, z\right)$ be the maximal conformal radius of $D_{n}$. Then

$$
\frac{R^{2}\left(D_{n}\right)}{\operatorname{area}\left(D_{n}\right)} \leq \frac{2^{4 / n}}{\pi} \frac{\Gamma\left(1-\frac{1}{n}\right) \Gamma\left(\frac{1}{2}+\frac{1}{n}\right)}{\Gamma\left(1+\frac{1}{n}\right) \Gamma\left(\frac{1}{2}-\frac{1}{n}\right)}
$$

with the sign of equality only for the regular Euclidean n-gons.

For $n=3,4$, (1.5) was proved by Pólya and Szegö [28], whose method based on the Steiner symmetrization fails for $n \geq 5$. Since at that time such problems were not accessible with known methods, the authors wrote in [28, p. 159] that "to prove (or disprove) the analogous theorems for the regular polygons with more than four sides is a challenging task". For $n \geq 5$, 1.5 was proved in [31.

As was noted above, every hyperbolic $n$-gon having the maximal $h$-area $\sigma_{n}=$ $\pi(n-2) / 4$ has all its angles equal to 0 and all its vertices lying on the ideal boundary $\mathbb{T}$. In this case, Theorem 1.1 leads to the following

Theorem $1.3(\sqrt{33})$. Let $D_{n} \ni 0$ be a hyperbolic polygon with $n \geq 3$ sides having all its vertices on $\mathbb{T}$. Then

$$
R_{h}\left(D_{n}, 0\right)=R\left(D_{n}, 0\right) \leq \frac{\Gamma\left(1-\frac{1}{n}\right) \Gamma\left(\frac{1}{2}+\frac{1}{n}\right)}{\Gamma\left(1+\frac{1}{n}\right) \Gamma\left(\frac{1}{2}-\frac{1}{n}\right)}
$$

with the sign of equality only for the regular hyperbolic $n$-gons centered at the origin. 
Inequality (1.6) was conjectured by J. Hersch in connection with his study of iteration of fundamental domains of certain Riemann surfaces; see [24]. For $n=3$, it was verified by R. Kühnau [24], while the general case was proved by Solynin [33].

The following two corollaries follow from (1.4) when $A \rightarrow 0^{+}$and when $n \rightarrow \infty$, respectively.

Corollary 1.1. Let $n \geq 3, A>0$. The following sharp inequalities hold for all hyperbolic polygons $D_{n}$ such that $h$-area $\left(D_{n}\right)=A$ :

$$
\frac{R_{h}^{2}\left(D_{n}\right)}{h \text {-area }\left(D_{n}\right)} \leq \frac{R_{h}^{2}\left(D_{n}(A)\right)}{A}<\frac{2}{\pi n} \frac{\Gamma\left(\frac{2}{n}\right) \Gamma^{2}\left(1-\frac{1}{n}\right)}{\Gamma\left(1-\frac{2}{n}\right) \Gamma^{2}\left(1+\frac{1}{n}\right)} .
$$

Corollary 1.2. Let $D \ni z_{0}$ be a simply connected domain in $\mathbb{U}$ such that $h$-area $(D)$ $=\pi \sinh ^{2} \rho$, where $\rho>0$. Then

$$
\frac{R_{h}^{2}\left(D, z_{0}\right)}{h \text {-area }(D)} \leq \frac{1}{\pi \cosh ^{2} \rho} \leq \frac{\rho^{2}}{\pi \sinh ^{2} \rho}<\frac{1}{\pi} .
$$

As we mentioned before, the regular hyperbolic $n$-gons maximize the $h$-area among all hyperbolic $n$-gons with a given hyperbolic perimeter and among all cyclic hyperbolic $n$-gons inscribed in a hyperbolic circle of a given radius. Thus Theorem 1.1, when combined with the latter properties, leads to the following two theorems.

Theorem 1.4. Let $D_{n} \ni z_{0}$ be a hyperbolic polygon with $n \geq 3$ sides and a fixed hyperbolic perimeter $L>0$. Then (1.4) holds with

$$
\beta=(1 / \pi) \sin ^{-1}(\cos (\pi / n) / \cosh (L / n))
$$

and $A=(\pi / 4)(n-2-2 \beta n)$ and with the sign of equality only for the regular $n$-gons centered at $z_{0}$.

Theorem 1.5. Let $D_{n} \ni z_{0}$ be a cyclic hyperbolic polygon with $n \geq 3$ sides inscribed in a hyperbolic circle centered at $z_{0}$ with the hyperbolic radius $\rho>0$. Then (1.4) holds with $\beta=(1 / \pi) \tan ^{-1}(\cot (\pi / n) / \cosh 2 \rho), A=(\pi / 4)(n-2-2 \beta n)$ and with the sign of equality only for the regular $n$-gons centered at $z_{0}$.

Note that for every $n \geq 3$ and $\rho>0$ there exist cyclic hyperbolic $n$-gons inscribed in a hyperbolic circle $C_{\rho}^{h}\left(z_{0}\right)=\left\{z: \rho\left(z, z_{0}\right)=\rho\right\}$ with the hyperbolic radius $\rho>0$ and center at $z_{0}$; see Figure 2 a. In contrast, for the hyperbolic $n$-gons circumscribed about $C_{\rho}^{h}\left(z_{0}\right)$, the situation is different: for $\rho>\rho_{n}$, where

$$
\rho_{n}=-(1 / 2) \log \tan (\pi / 2 n)
$$

there are no hyperbolic $n$-gons circumscribed about $C_{\rho}^{h}\left(z_{0}\right)$. For $\rho=\rho_{n}$, the regular hyperbolic $n$-gons centered at $z_{0}$ are the only hyperbolic $n$-gons circumscribed about $C_{\rho}^{h}\left(z_{0}\right)$; see Figure $2 \mathrm{~b}$. This is another remarkable difference between the hyperbolic and Euclidean geometries.

The situation is also changed when we deal with another aspect of the main problem of this paper: for $\rho<\rho_{n}$ the regular $n$-gons do not maximize the conformal $h$-radius over the set of all hyperbolic $n$-gons circumscribed about $C_{\rho}^{h}\left(z_{0}\right)$. In contrast, they provide the minimal value for the conformal $h$-radius. Note that the radius of the maximal hyperbolic disc embedded in a Riemann surface $\mathcal{R}$, known as the injectivity radius of $\mathcal{R}$, is an important characteristic of $\mathcal{R}$. From this point of view, our next two theorems give inequalities linking the injectivity radius of 

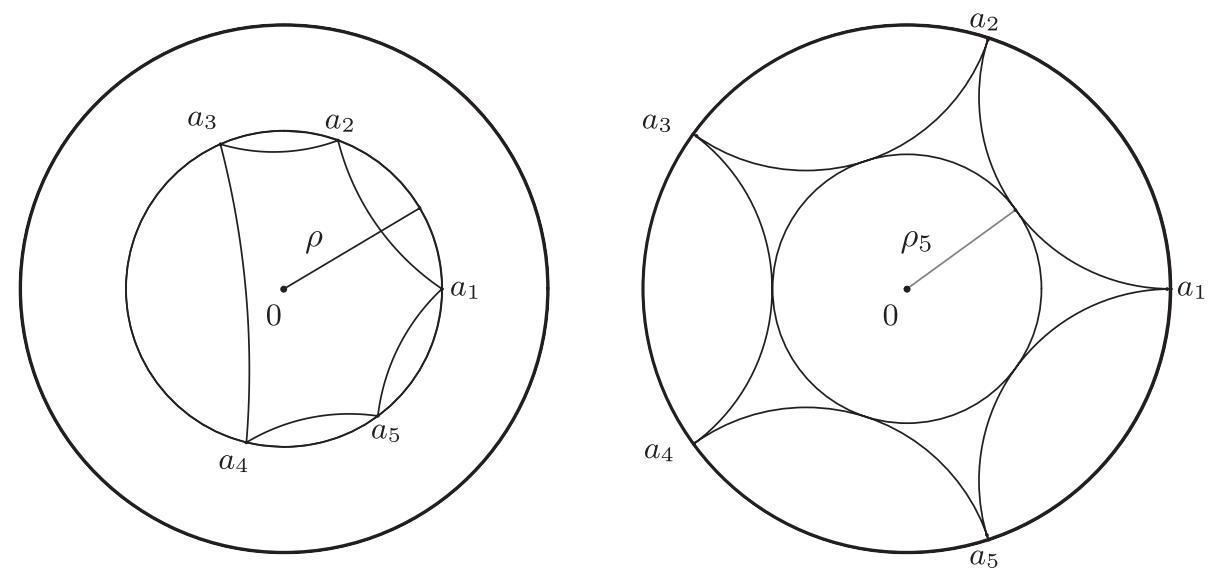

a) Cyclic 5-gon in a circle of $h$-radius $\rho$ b) Regular 5-gon circumscribed about $C_{\rho_{5}}^{h}(0)$.

Figure 2. Cyclic and circumcsribed polygons

a Riemann surface, the conformal $h$-radius of its fundamental polygon, and the number of sides of the polygon.

Theorem 1.6. For $0<\rho<\rho_{n}$, let $D_{n}$ be a hyperbolic $n$-gon with $n \geq 3$ sides circumscribed about $C_{\rho}^{h}\left(z_{0}\right)$ and let $\beta=(1 / \pi) \cos ^{-1}(\sin (\pi / n) \cosh (2 \rho))$. Then

$$
R_{h}^{2}\left(D_{n}, z_{0}\right) \geq \frac{\Gamma^{2}\left(1-\frac{1}{n}\right) \Gamma\left(\frac{1}{2}+\frac{1}{n}+\beta\right) \Gamma\left(\frac{1}{2}+\frac{1}{n}-\beta\right)}{\Gamma^{2}\left(1+\frac{1}{n}\right) \Gamma\left(\frac{1}{2}-\frac{1}{n}+\beta\right) \Gamma\left(\frac{1}{2}-\frac{1}{n}-\beta\right)},
$$

with the sign of equality only for the regular $n$-gons circumscribed about $C_{\rho}^{h}\left(z_{0}\right)$.

Theorem 1.6 gives the sharp lower bound for the conformal $h$-radius among all polygons circumscribed about $C_{\rho}^{h}\left(z_{0}\right)$, which have a fixed number of sides. In Theorem 1.7 we show that without a restriction on the number of sides, the regular $n$-gon circumscribed about $C_{\rho_{n}}^{h}\left(z_{0}\right)$ maximizes the conformal $h$-radius in the family of all hyperbolic polygons circumscribed about $C_{\rho}^{h}\left(z_{0}\right)$ with $0<\rho \leq \rho_{n}$, where $\rho_{n}$ are the discrete values defined by (1.9).

Theorem 1.7. For $0<\rho \leq \rho_{n}$, let $D$ be a hyperbolic polygon circumscribed about $C_{\rho}^{h}\left(z_{0}\right)$. Then

$$
R_{h}\left(D, z_{0}\right) \leq \frac{\Gamma\left(1-\frac{1}{n}\right) \Gamma\left(\frac{1}{2}+\frac{1}{n}\right)}{\Gamma\left(1+\frac{1}{n}\right) \Gamma\left(\frac{1}{2}-\frac{1}{n}\right)}
$$

with the sign of equality only for the regular hyperbolic n-gons circumscribed about $C_{\rho_{n}}^{h}\left(z_{0}\right)$.

The proof of Theorem 1.1 and its corollaries is given in Section 3, where we use the method developed in [31]-36] based on a special triangulation of a polygon. Section 2 contains necessary prerequisites. The proof consists of two main parts. First we show that the quantity $m_{h}\left(D_{n}, z_{0}\right)=(1 / 2 \pi) \log R_{h}\left(D_{n}, z_{0}\right)$, called the reduced $h$-module of $D_{n}$ at $z_{0}$, admits an explicit upper bound given by a weighted sum of the reduced $h$-modules of triangles composing $D_{n}$, each of which has a distinguished vertex at $z_{0}$. The precise definitions and formulations will be given 
in Section 2. This explicit bound is a complicated combination of the gamma functions. The study of such special functions is of independent interest. There is a huge literature devoted to them [2, 3, 4]. We shall use the following theorem, the proof of which is given in Section 6 . Let

$$
F(\alpha, \beta)=\alpha \log \frac{\Gamma^{2}(1-\alpha) \Gamma\left(\frac{1}{2}+\alpha+\beta\right) \Gamma\left(\frac{1}{2}+\alpha-\beta\right)}{\Gamma^{2}(1+\alpha) \Gamma\left(\frac{1}{2}-\alpha+\beta\right) \Gamma\left(\frac{1}{2}-\alpha-\beta\right)} .
$$

Theorem 1.8. The function $F(\alpha, \beta)$ is strictly concave in the simplex $\Pi=\{(\alpha, \beta)$ : $\alpha>0, \beta \geq 0, \alpha+\beta<1 / 2\}$, i.e.

$$
\sum_{k=1}^{m} F\left(\alpha_{k}, \beta_{k}\right) \leq m F\left(\alpha_{0}, \beta_{0}\right)
$$

for every positive integer $m$, every $\left(\alpha_{k}, \beta_{k}\right) \in \Pi, k=1, \ldots, m$, and

$$
\alpha_{0}=\frac{1}{m} \sum_{k=1}^{m} \alpha_{k}, \quad \beta_{0}=\frac{1}{m} \sum_{k=1}^{m} \beta_{k} .
$$

Equality in (1.13) occurs if and only if $\left(\alpha_{k}, \beta_{k}\right)=\left(\alpha_{0}, \beta_{0}\right)$ for all $k=1, \ldots, m$.

Theorems 1.6 and 1.7 are proved in Section 4. The proof of Theorem 1.7 also uses the decomposition of $D_{n}$ into triangles. Here it is interesting to note that, by decomposing a given polygon into triangles, we reduced the problem in Theorem 1.1 to the maximization problem for a concave function, which allows us to increase the number of sides of a polygon if necessary. In contrast, the problem in Theorem 1.7 is reduced to the maximization problem for a convex function, which shows that the extremal polygon must have a minimal possible number of sides.

The proof of Theorem 1.6 uses a very different approach from the proofs of our other theorems. It requires the method of dissymmetrization, invented by V. N. Dubinin [10] in the form used in 32]. Section 4 also contains the necessary definitions and preliminary results concerning dissymmetrization.

An important observation is that although all the theorems in this paper are formulated for simply connected polygonal domains, our proofs lead to much stonger results. This situation is typical for the method of decomposition into triangles, as was emphasized in [31, 32, 34, 35]. To be more precise, let $\mathcal{R}$ be a simply connected Riemann surface over the complex sphere $\overline{\mathbb{C}}$ and let $\mathcal{R}_{z_{0}}^{*}$ be the star of $\mathcal{R}$ with respect to the point $z=z_{0}$, i.e. $\mathcal{R}_{z_{0}}^{*}$ is the largest simply connected planar domain in $\mathcal{R}$ containing the point $z_{0}$ and such that the straight line segment $\left[z_{0}, z\right]$ belongs to $\mathcal{R}_{z_{0}}^{*}$ if $z$ does. A simply connected domain $D$ is called starlike with respect to $z_{0} \in D$ if $D_{z_{0}}^{*}=D$. A polygon $D \ni 0$ (on the Euclidean or hyperbolic plane) is called $m$-star shaped with respect to $z=0$ if it is starlike with respect to $z=0$ and if segments joining $z=0$ with the vertices of $D$ split it into $m \geq 3$ triangles (Euclidean or hyperbolic). The $m$-star shapeness of $D$ with respect to an arbitrary point $z_{0} \in D$ is defined in a similar way.

Inequality (1.4) holds with $D_{n}$ replaced by $\mathcal{R}$ for any simply connected Riemann surface $\mathcal{R}$ described above if its hyperbolic star $\mathcal{R}_{z_{0}}^{*}$ is contained in an $n$-star shaped hyperbolic polygon having hyperbolic area $A$. Our other inequalities (except inequality (1.10), the proof of which uses a different method based on dissymmetrization) admit similar generalizations.

This research was motivated by the work of Solynin in the area of extremal problems for certain conformal invariants 31, 33, 34, 35. It consists of two parts. 
The first part deals with the extremal problems properly. The second part includes results concerning properties of special functions necessary for the main proofs of the first part, which also present significant interest in their own right.

\section{HyPERBOLIC TRIGONOMETRY AND MODULES}

The conformal radius $R\left(D, z_{0}\right)$ of a simply connected domain $D$ is related to another characteristic of $D$, the so-called reduced module of $D$ at $z_{0}$ introduced by O. Teichmüller; see [21, p. 24]. Since all the domains we are working with are on the hyperbolic plane, it is convenient to use a variant called the reduced $h$-module defined as follows.

Let $D \ni z_{0}$ be a simply connected domain on $\mathbb{U}$. For $\varepsilon>0$ small enough, let $D_{\varepsilon}$ denote the doubly-connected domain having the boundary components $\partial D$ and $C_{\varepsilon}^{h}\left(z_{0}\right)$. Let $\bmod \left(D_{\varepsilon}\right)$ denote the module of $D_{\varepsilon}$ for the class of closed Jordan curves separating the boundary components of $D_{\varepsilon}$; see [21, Ch.2]. Then the limit

$$
m_{h}\left(D, z_{0}\right)=\lim _{\varepsilon \rightarrow 0^{+}}\left(\bmod \left(D_{\varepsilon}\right)+(1 / 2 \pi) \log \varepsilon\right),
$$

which exists and is finite, will be called the reduced h-module (hyperbolic module) of $D$ at $z_{0}$. The only difference compared to the standard definition of the reduced module $m\left(D, z_{0}\right)$ [21, p. 24] is that we use the hyperbolic radius of the removed disk instead of the Euclidean radius.

The equality

$$
m_{h}\left(D, z_{0}\right)=m\left(D, z_{0}\right)-(1 / 2 \pi) \log \left(1-\left|z_{0}\right|^{2}\right)
$$

links the hyperbolic and Euclidean reduced modules. The advantage of using the reduced $h$-module in hyperbolic geometry is its invariance under isometries of the hyperbolic plane. The reduced $h$-module and conformal $h$-radius are related via the formula

$$
m_{h}(D, z)=(1 / 2 \pi) \log R_{h}(D, z),
$$

which shows, in particular, that (1.7) holds if and only if $D_{n}(A)$ maximizes the reduced $h$-module $m_{h}\left(D_{n}, z_{0}\right)$ among all hyperbolic $n$-gons $D_{n}$ such that $h$-area $\left(D_{n}\right)$ $=A$.

As was mentioned in the Introduction, our approach to the considered problems is based on a special triangulation of $D_{n}$. By a trilateral $D=D\left(e_{1}, e_{2}, e_{3}\right)$ we shall mean a simply connected domain $D$ (in general lying on a Riemann surface) with three distinct points $e_{1}, e_{2}, e_{3}$ (called vertices) on its boundary. For the purposes of this paper, it is enough to deal with trilaterals on $\mathbb{U}$ having a piecewise smooth Jordan boundary such that $e_{1} \in \mathbb{U}$ and $l_{\varepsilon}=D \cap C_{\varepsilon}^{h}\left(e_{1}\right)$ is connected for all $\varepsilon>0$ small enough. Under our assumptions, $e_{2}, e_{3} \in \overline{\mathbb{U}}$. Let $D_{\varepsilon}\left(e_{1}\right)=D \backslash \overline{\mathbb{U}_{\varepsilon}^{h}}\left(e_{1}\right)$. Here $\mathbb{U}_{\varepsilon}^{h}\left(z_{0}\right)=\left\{z: \rho\left(z, z_{0}\right)<\varepsilon\right\}$ is a hyperbolic disc centered at $z_{0} \in \mathbb{U}$ having hyperbolic radius $\varepsilon$. Considering $D_{\varepsilon}\left(e_{1}\right)$ as a quadrilateral with distinguished sides $\overbrace{2} e_{3}$ and $l_{\varepsilon}$, let $\bmod \left(D_{\varepsilon}\left(e_{1}\right)\right)$ denote the module of $D_{\varepsilon}\left(e_{1}\right)$ for the class of curves separating $e_{2} e_{3}$

from $l_{\varepsilon}$ in $D_{\varepsilon}\left(e_{1}\right)$; see [21, Ch. 2]. Let $D$ have an inner angle $\varphi \in(0,2 \pi]$ at $e_{1}$. The limit

$$
m_{h}\left(D ; e_{1} \mid e_{2}, e_{3}\right)=\lim _{\varepsilon \rightarrow 0^{+}}\left(\bmod \left(D_{\varepsilon}\left(e_{1}\right)\right)+(1 / \varphi) \log \varepsilon\right),
$$

provided it is well defined and finite, is called the reduced $h$-module of $D$ at $e_{1}$. This definition is a hyperbolic version of the notion of the reduced module $m\left(D ; e_{1} \mid e_{2}, e_{3}\right)$ 
of a trilateral introduced in 31]. The equality

$$
m_{h}\left(D ; e_{1} \mid e_{2}, e_{3}\right)=m\left(D ; e_{1} \mid e_{2}, e_{3}\right)-(1 / \varphi) \log \left(1-\left|e_{1}\right|^{2}\right)
$$

(cf. (2.1) ) links the hyperbolic and Euclidean reduced modules of $D$. The finite limit in (2.3) may not exist even if $D$ has a piecewise analytic boundary; cf. 35. Example 1.1]. In [35] some sufficient conditions for the existence of $m\left(D ; e_{1} \mid e_{2}, e_{3}\right)$, or equivalently for the existence of $m_{h}\left(D ; e_{1} \mid e_{2}, e_{3}\right)$, are given. In this paper we deal only with trilaterals bounded by circular arcs, which guarantees existence of all the reduced modules considered below.

It is clear from the definition that the reduced $h$-module of a trilateral is invariant under isometries of the hyperbolic plane. For an arbitrary conformal mapping $f: D \rightarrow \mathbb{U}$, the change in the reduced $h$-module is characterized by

Lemma 2.1. Let $f$ conformally map a trilateral $D=D\left(e_{1}, e_{2}, e_{3}\right) \subset \mathbb{C}$ having an angle $0<\varphi \leq 2 \pi$ at the vertex $e_{1} \in \mathbb{C}$ onto a trilateral $D_{f}\left(\zeta_{1}, \zeta_{2}, \zeta_{3}\right) \subset \mathbb{U}$ such that $f\left(e_{k}\right)=\zeta_{k}$ for $k=1,2,3$, and $\zeta_{1} \in \mathbb{U}$. Assume that for all $z \in D$ in a vicinity of the vertex $e_{1}$,

$$
f(z)=\zeta_{1}+C\left(z-e_{1}\right)^{\alpha}(1+g(z))
$$

where $C \in \mathbb{C}, C \neq 0,0<\alpha \varphi \leq 2 \pi, g$ is continuous in a vicinity of $e_{1}$, and $g\left(e_{1}\right)=0$. Then

$$
m_{h}\left(D_{f} ; \zeta_{1} \mid \zeta_{2}, \zeta_{3}\right)=m\left(D ; e_{1} \mid e_{2}, e_{3}\right)+\frac{1}{\alpha \varphi} \log \frac{|C|}{1-\left|\zeta_{1}\right|^{2}} .
$$

In addition, if $D \subset \mathbb{U}$ and $e_{1} \in \mathbb{U}$, then

$$
m_{h}\left(D_{f} ; \zeta_{1} \mid \zeta_{2}, \zeta_{3}\right)=m_{h}\left(D ; e_{1} \mid e_{2}, e_{3}\right)+\frac{1}{\alpha \varphi} \log \frac{|C|\left(1-\left|e_{1}\right|^{2}\right)^{\alpha}}{1-\left|\zeta_{1}\right|^{2}}
$$

Equation (2.5) easily follows from the definition of the reduced $h$-module or, via (2.4), from a similar result for Euclidean reduced modules, [31, 35.

Lemma 2.1 and equality (2.1), linking the hyperbolic and Euclidean reduced modules, allow us to compute the reduced $h$-modules using a conformal mapping onto certain canonical trilaterals such as the circular sector $S_{R}(\alpha)=\{z:|z|<$ $R, 0<\arg z<\alpha\}, R>0,0<\alpha \leq 2 \pi$, with the vertices $e_{1}=0, e_{2}=R, e_{3}=R e^{i \alpha}$ and the upper half-plane $\mathbb{H}=\{z: \Im z>0\}$ with the vertices $e_{1}=0, e_{2}=R$, $e_{3}=\infty$. For these two configurations, one can find from the definition,

$$
m\left(S_{R}(\alpha) ; 0 \mid R, R e^{i \alpha}\right)=(1 / \alpha) \log R, \quad m(\mathbb{H} ; 0 \mid R, \infty)=(1 / \pi) \log 4 R .
$$

Let $D^{1}, D^{2}, \ldots, D^{n}$ be pairwise disjoint trilaterals in a simply connected domain $D \subset \mathbb{U}$ such that each $D^{k}$ has a vertex $e_{1}^{k}$ at the point $z_{0} \in D$ and the opposite side $e_{2}^{\widehat{k} e_{3}^{k}}$ on $\partial D$; see Figure 3 Note that $\bigcup_{k=1}^{n} D^{k} \subset D$, and $z_{0}=e_{1}^{k}$ for $k=$ $1, \ldots, n$. The next result linking the reduced $h$-module of $D$ at $z_{0}$ with the reduced modules of the trilaterals $D^{k}$ composing $D$, which is a reformulation of Theorem 3 in [31, is basic for our further considerations.

Theorem 2.1 (cf. 31, 35). Let $D^{k}$ have an angle $0<2 \alpha_{k} \pi \leq 2 \pi$ at the vertex $e_{1}^{k}$ and assume that for every $k=1, \ldots, n$, the reduced $h$-module of $D^{k}$ at $e_{1}^{k}=z_{0}$ exists. If $\sum_{k=1}^{n} \alpha_{k}=1$, then

$$
m_{h}\left(D, z_{0}\right) \leq \sum_{k=1}^{n} \alpha_{k}^{2} m_{h}\left(D^{k} ; z_{0} \mid e_{2}^{k}, e_{3}^{k}\right)
$$




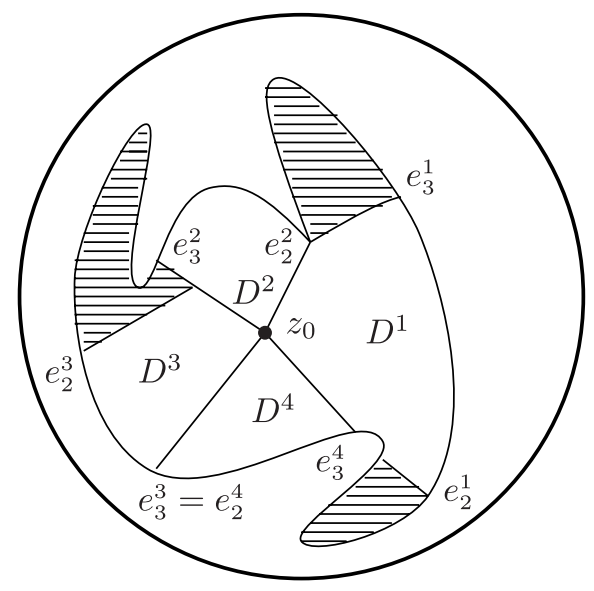

Figure 3. Decomposition of a domain $D$ into trilaterals

Let $f$ be a conformal mapping from $\mathbb{U}$ onto $D$ such that $f(0)=z_{0}$. Equality occurs in (2.7) if and only if for every $k=1, \ldots, n, f^{-1}\left(D^{k}\right)$ is a sector of $\mathbb{U}$ of opening $2 \pi \alpha_{k}$, where the geometric vertices of the sector correspond under the mapping $f$ to the vertices of $D^{k}$.

Inequality (2.7) follows from the well-known subadditivity property of the module (or equivalently of the extremal length) of a family of curves; see [1, 21, 35]. Theorem 2.1]admits some useful extensions. For instance, we can consider pairwise disjoint trilaterals $D^{k}=D^{k}\left(e_{1}^{k}, e_{2}^{k}, e_{3}^{k}\right), k=1,2, \ldots, n$, lying in a larger trilateral $D=D\left(e_{1}, e_{2}, e_{3}\right)$ such that $D^{k}$ has the vertex $e_{1}^{k}$ at $e_{1}$ and the opposite side $e_{2}^{k} e_{3}^{k}$ on the side $e_{2} e_{3}$ of $D$. Then we have

Theorem 2.2 (cf. 31, 35). Let $D$ and $D^{k}$ have the angles $2 \beta \pi$ and $2 \alpha_{k} \beta \pi$, where $0<2 \alpha_{k} \beta \pi \leq 2 \beta \pi \leq 2 \pi$, at their vertices $e_{1}$ and $e_{1}^{k}$, respectively. If all the reduced modules considered below exist and if $\sum_{k=1}^{n} \alpha_{k}=1$, then

$$
m_{h}\left(D ; e_{1} \mid e_{2}, e_{3}\right) \leq \sum_{k=1}^{n} \alpha_{k}^{2} m_{h}\left(D^{k} ; e_{1}^{k} \mid e_{2}^{k}, e_{3}^{k}\right) .
$$

Let $f$ be a conformal mapping from the circular sector $S_{1}(2 \beta \pi)$ onto $D$, which sends the geometric vertices of the sector to the vertices of $D$ such that $f(0)=e_{1}$. Equality occurs in (2.8) if and only if for every $k=1, \ldots, n, f^{-1}\left(D^{k}\right)$ is a sector in $S_{1}(2 \beta \pi)$ of opening $2 \alpha_{k} \beta \pi$, where the geometric vertices of the sector correspond under the mapping $f$ to the vertices of $D^{k}$.

Now we consider an instructive example that is important for what then follows. From this position all the considered trilaterals will be hyperbolic triangles having their geometric vertices as the distinguished boundary points. In this case we prefer the term "triangle" rather than "trilateral".

Let $T=T(\alpha, \beta, \gamma)$ be a hyperbolic triangle having the angles $\alpha \pi, \beta \pi$, and $\gamma \pi$ at the vertices $e_{1} \in \mathbb{U}$ and $e_{2}, e_{3} \in \overline{\mathbb{U}}$, respectively. Since the reduced $h$-module is invariant under the isometries of the hyperbolic plane we shall assume that $e_{1}=0$, $e_{2}=r$ where $0<r \leq 1$, and $\Im e_{3}>0$; see Figure 4. For convenience, we shall assume also that $\beta \leq \gamma$. 


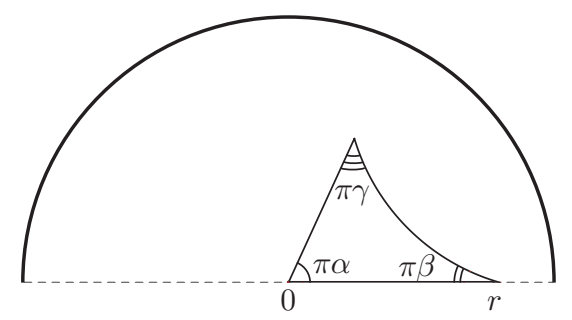

Figure 4. Triangle $T(\alpha, \beta, \gamma)$

If $\beta=0$, then $r=1$. To compute $r$ in the case $\beta>0$, we apply the second cosine theorem of hyperbolic geometry (see [6, p.148]),

$$
\cosh 2 c=\frac{\cos \alpha \pi \cos \beta \pi+\cos \gamma \pi}{\sin \alpha \pi \sin \beta \pi},
$$

where $c$ denotes the hyperbolic distance between $e_{1}=0$ and $e_{2}=r$. We remind the reader that the appearance of the factor 2 in (2.9) is due to our choice of the hyperbolic metric (1.1). Since $c=(1 / 2) \log ((1+r) /(1-r))>0$, we have

$$
r=\tanh c=\sqrt{\frac{\cosh 2 c-1}{\cosh 2 c+1}}
$$

From (2.9) and (2.10), we obtain

$$
r^{2}=\frac{\cos (\pi(\alpha+\beta+\gamma) / 2) \cos (\pi(\alpha+\beta-\gamma) / 2)}{\cos (\pi(\alpha-\beta+\gamma) / 2) \cos (\pi(\alpha-\beta-\gamma) / 2)}
$$

Using the reflection formula [5, p. 3],

$$
\Gamma(1 / 2+z) \Gamma(1 / 2-z)=\pi / \cos \pi z,
$$

we can express $r$ in terms of the gamma functions:

$$
\begin{aligned}
r^{2}= & \frac{\Gamma\left(\frac{1}{2}+\frac{1}{2}(\alpha-\beta+\gamma)\right) \Gamma\left(\frac{1}{2}-\frac{1}{2}(\alpha-\beta+\gamma)\right)}{\Gamma\left(\frac{1}{2}+\frac{1}{2}(\alpha+\beta+\gamma)\right) \Gamma\left(\frac{1}{2}-\frac{1}{2}(\alpha+\beta+\gamma)\right)} \\
& \times \frac{\Gamma\left(\frac{1}{2}+\frac{1}{2}(\alpha-\beta-\gamma)\right) \Gamma\left(\frac{1}{2}-\frac{1}{2}(\alpha-\beta-\gamma)\right)}{\Gamma\left(\frac{1}{2}+\frac{1}{2}(\alpha+\beta-\gamma)\right) \Gamma\left(\frac{1}{2}-\frac{1}{2}(\alpha+\beta-\gamma)\right)} .
\end{aligned}
$$

To find $m_{h}\left(T ; 0 \mid e_{2}, e_{3}\right)$, we consider the function

$$
z=f_{0}(\zeta)=c \frac{(-\zeta)^{\alpha} F\left(\frac{1}{2}+\frac{1}{2}(\alpha-\beta-\gamma), \frac{1}{2}+\frac{1}{2}(\alpha-\beta+\gamma) ; 1+\alpha ; \zeta\right)}{F\left(\frac{1}{2}-\frac{1}{2}(\alpha+\beta+\gamma), \frac{1}{2}-\frac{1}{2}(\alpha+\beta-\gamma) ; 1-\alpha ; \zeta\right)},
$$

where $-\pi<\arg (-\zeta)<0, F(\cdot, \cdot ; \cdot ; \cdot)$ is the Gauss hypergeometric function defined by its expansion for $|\zeta|<1$, and

$$
c=r e^{i \alpha \pi} \frac{\Gamma(1-\alpha) \Gamma\left(\frac{1}{2}+\frac{1}{2}(\alpha+\beta+\gamma)\right) \Gamma\left(\frac{1}{2}+\frac{1}{2}(\alpha+\beta-\gamma)\right)}{\Gamma(1+\alpha) \Gamma\left(\frac{1}{2}-\frac{1}{2}(\alpha-\beta-\gamma)\right) \Gamma\left(\frac{1}{2}-\frac{1}{2}(\alpha-\beta+\gamma)\right)} .
$$

The function $f_{0}$ maps the upper half plane $\mathbb{H}$ onto the triangle $T=T(\alpha, \beta, \gamma)$ such that $f_{0}(0)=0, f_{0}(1)=r$, and

$$
f_{0}(\infty)=r e^{i \alpha \pi} \frac{\cos (\pi(\alpha-\beta+\gamma) / 2)}{\cos (\pi(\alpha+\beta-\gamma) / 2)}
$$


see [17, Sections 9.151-9.153] or [22, pp. 229-230] for a detailed discussion. Equations (2.12) and (2.13) lead to the asymptotic equality

$$
f_{0}(\zeta)=c(-\zeta)^{\alpha}(1+g(\zeta)) \quad \text { as } \quad \zeta \rightarrow 0
$$

where $g$ is continuous in a vicinity of $\zeta=0$ and $g(0)=0$. Using Lemma 2.1 relations (2.13), (2.14), and the second equality in (2.6), we obtain, following [33], $(2.15)$

$$
m_{h}\left(T ; 0 \mid e_{2}, e_{3}\right)=\frac{1}{\alpha \pi} \log \frac{4^{\alpha} r \Gamma(1-\alpha) \Gamma\left(\frac{1}{2}+\frac{1}{2}(\alpha+\beta+\gamma)\right) \Gamma\left(\frac{1}{2}+\frac{1}{2}(\alpha+\beta-\gamma)\right)}{\Gamma(1+\alpha) \Gamma\left(\frac{1}{2}-\frac{1}{2}(\alpha-\beta-\gamma)\right) \Gamma\left(\frac{1}{2}-\frac{1}{2}(\alpha-\beta+\gamma)\right)} .
$$

Substituting $r$ from (2.11) into (2.15), we obtain the desired formula for the reduced $h$-module of $T$ :

$$
\begin{aligned}
m_{h}\left(T ; 0 \mid e_{2}, e_{3}\right)= & \frac{1}{2 \alpha \pi} \log \left[\frac{4^{2 \alpha} \Gamma^{2}(1-\alpha)}{\Gamma^{2}(1+\alpha)}\right. \\
& \times \frac{\Gamma\left(\frac{1}{2}+\frac{1}{2}(\alpha+\beta+\gamma)\right) \Gamma\left(\frac{1}{2}+\frac{1}{2}(\alpha+\beta-\gamma)\right)}{\Gamma\left(\frac{1}{2}-\frac{1}{2}(\alpha+\beta+\gamma)\right) \Gamma\left(\frac{1}{2}-\frac{1}{2}(\alpha+\beta-\gamma)\right)} \\
& \left.\times \frac{\Gamma\left(\frac{1}{2}+\frac{1}{2}(\alpha-\beta+\gamma)\right) \Gamma\left(\frac{1}{2}+\frac{1}{2}(\alpha-\beta-\gamma)\right)}{\Gamma\left(\frac{1}{2}-\frac{1}{2}(\alpha-\beta+\gamma)\right) \Gamma\left(\frac{1}{2}-\frac{1}{2}(\alpha-\beta-\gamma)\right)}\right]
\end{aligned}
$$

It turns out that the reduced modules studied in this paper can be expressed as combinations of the gamma function for different values of the variable. To unify notation, it is convenient to employ the function

$$
S(a, b, c, t)=c \log \frac{\Gamma^{2}(1-a) \Gamma(1 / 2+a+b t) \Gamma(1 / 2+a-b t)}{\Gamma^{2}(1+a) \Gamma(1 / 2-a+b t) \Gamma(1 / 2-a-b t)} .
$$

With this notation, the function $F$ defined by (1.12) becomes $F(\alpha, \beta)=S(\alpha, \beta, \alpha, 1)$. For $\alpha>0, \beta \geq 0$ such that $\alpha+\beta<1 / 2$ and $0 \leq t \leq 1$, the reduced $h$-module of the triangle $T=T(2 \alpha, \beta(1-t), \beta(1+t))$ can be expressed as

$$
m_{h}\left(T ; 0 \mid e_{2}, e_{3}\right)=C_{0}+S\left(\alpha, \beta,(4 \alpha \pi)^{-1}, t\right),
$$

where

$$
C_{0}=\frac{1}{4 \alpha \pi} \log \frac{4^{4 \alpha} \Gamma^{2}(1-2 \alpha) \Gamma^{2}(1+\alpha) \Gamma\left(\frac{1}{2}+\alpha+\beta\right) \Gamma\left(\frac{1}{2}+\alpha-\beta\right)}{\Gamma^{2}(1+2 \alpha) \Gamma^{2}(1-\alpha) \Gamma\left(\frac{1}{2}-\alpha+\beta\right) \Gamma\left(\frac{1}{2}-\alpha-\beta\right)}
$$

does not depend on $t$.

Note that for fixed $\alpha \in(0,1 / 2)$ and $A \in(0, \pi(1-2 \alpha) / 4)$ and for $\beta=1 / 2-\alpha-$ $2 A / \pi$, all the triangles $T(2 \alpha, \beta(1-t), \beta(1+t))$ have the same hyperbolic area $A$ independent of $t$.

For the isosceles triangle $T(2 \alpha, \beta, \beta)$ and for the right triangle $T(\alpha, \beta, 1 / 2),(2.16)$ gives

$$
\begin{aligned}
m_{h}\left(T(2 \alpha, \beta, \beta) ; 0 \mid e_{2}^{i}, e_{3}^{i}\right) & =(1 / 2) m_{h}\left(T(\alpha, \beta, 1 / 2) ; 0 \mid e_{2}^{r}, e_{3}^{r}\right) \\
& =\frac{1}{4 \alpha^{2} \pi} F(\alpha, \beta)=S\left(\alpha, \beta,(4 \alpha \pi)^{-1}, 1\right),
\end{aligned}
$$

where $e_{k}^{i}$ and $e_{k}^{r}, k=2,3$, are the vertices of the corresponding triangle different from $z=0$. 
Lemma 2.2. For fixed $\alpha, A$, and $\beta>0$ as above, let $F_{1}(t)$ denote the reduced $h$-module $m_{h}\left(T ; 0 \mid e_{2}, e_{3}\right)$ of $T=T(2 \alpha, \beta(1-t), \beta(1+t))$ considered as a function of $t$. Then $F_{1}(t)$ is an even, strictly concave, function in $-1 \leq t \leq 1$. In particular,

$$
F_{1}(t)<F_{1}(0)=\frac{1}{4 \alpha^{2} \pi} F(\alpha, \beta)=S\left(\alpha, \beta,(4 \alpha \pi)^{-1}, 1\right)
$$

for all $t \neq 0$.

The inequality in (2.19) shows that the isosceles triangle $T(2 \alpha, \beta, \beta)$ has the maximal reduced module among all hyperbolic triangles with a fixed angle $2 \alpha \pi$ and prescribed hyperbolic area $A$.

Lemma 2.2 will follow from Lemma 5.1 in Section 5 , where we will show that $S(a, b, c, t)$ is an absolutely monotonic function on $0<t<1$ for fixed $a, b>0$ and $c<0$ with $a+b<1 / 2$.

For $n \geq 3$ and $A \in\left(0, \pi(n-2) / 4\right.$ ], let $T_{n}(A)=T(2 / n, \beta, \beta)$ with $\beta=1 / 2-$ $1 / n-2 A / \pi n$. Then $T_{n}(A)$ is one of the $n$ equal isosceles triangles composing the regular $n$-gon $D_{n}(A)$. Equation (2.16) with $\alpha=2 / n$ and $\gamma=\beta$ gives

$m_{h}\left(T_{n}(A) ; 0 \mid e_{2}, e_{3}\right)=\frac{n^{2}}{4 \pi} F(1 / n, \beta)=\frac{n}{4 \pi} \log \frac{\Gamma^{2}\left(1-\frac{1}{n}\right) \Gamma\left(\frac{1}{2}+\frac{1}{n}+\beta\right) \Gamma\left(\frac{1}{2}+\frac{1}{n}-\beta\right)}{\Gamma^{2}\left(1+\frac{1}{n}\right) \Gamma\left(\frac{1}{2}-\frac{1}{n}-\beta\right) \Gamma\left(\frac{1}{2}-\frac{1}{n}+\beta\right)}$.

The latter relation combined with the assertion on the case of equality in Theorem 2.1 leads to the following expression for the reduced $h$-module of the regular polygon $D_{n}(A)$ :

(2.20) $m_{h}\left(D_{n}(A), 0\right)=(n / 4 \pi) F(1 / n, \beta)=S(1 / n, 1 / 2-(1 / n)(1+2 A / \pi), 1 / 4 \pi, 1)$.

The next lemma treats $m_{h}\left(D_{n}(A), 0\right)$ as a function of the number of sides of $D_{n}(A)$.

Lemma 2.3. For a fixed hyperbolic area $A$, the reduced h-module $m_{h}\left(D_{n}(A), 0\right)$ is defined and is strictly increasing in $n$ for $n \geq 2+4 A / \pi$.

This lemma will follow from Lemma 5.2 in Section 5, where we show that for fixed $c>0$ and $\omega>1$ the function $S(a, 1 / 2-a \omega,-c, 1)$ is absolutely monotonic for $0<a<1 /(1+\omega)$.

\section{Proof of the isoperimetric inequalities}

Proof of Theorem 1.1. For $n \geq 3$, let $D_{n}$ be a hyperbolic $n$-gon having hyperbolic area $A$, and let $z_{0} \in D_{n}$. Since the reduced $h$-module and hyperbolic area are invariant under the isometries of the hyperbolic plane, we may assume that $z_{0}=0$. Let $D_{n}^{*}$ denote the hyperbolic star of $D_{n}$ with respect to the origin. The rectilinear segments joining the origin with the geometric vertices of $D_{n}^{*}$ split $D_{n}^{*}$ into $m$ hyperbolic triangles $T_{k}, k=1,2, \ldots, m$, where $3 \leq m \leq n$. Let $2 \alpha_{k} \pi$ denote the angle of $T_{k}$ at the vertex $z=0$. Then $\sum_{k=1}^{m} \alpha_{k}=1$. The system of the triangles $T_{k}, k=1,2, \ldots, m$, is a competing system of trilaterals for the domain $D_{n}$ in the sense of Theorem 2.1. Therefore that theorem implies

$$
m_{h}\left(D_{n}, 0\right) \leq \sum_{k=1}^{m} \alpha_{k}^{2} m_{h}\left(T_{k} ; 0 \mid e_{2}^{k}, e_{3}^{k}\right)
$$

where $e_{2}^{k}$ and $e_{3}^{k}$ are the geometric vertices of $T_{k}$ different from the origin.

Let

$$
s_{k}=h \text {-area }\left(T_{k}\right), \quad \beta_{k}=1 / 2-\alpha_{k}-2 s_{k} / \pi .
$$


Then,

$$
A^{*}=\sum_{k=1}^{m} s_{k} \leq A .
$$

By Lemma 2.2

$$
m_{h}\left(T_{k} ; 0 \mid e_{2}^{k}, e_{3}^{k}\right) \leq \frac{1}{4 \alpha_{k} \pi} \log \frac{\Gamma^{2}\left(1-\alpha_{k}\right) \Gamma\left(\frac{1}{2}+\alpha_{k}+\beta_{k}\right) \Gamma\left(\frac{1}{2}+\alpha_{k}-\beta_{k}\right)}{\Gamma^{2}\left(1+\alpha_{k}\right) \Gamma\left(\frac{1}{2}-\alpha_{k}-\beta_{k}\right) \Gamma\left(\frac{1}{2}-\alpha_{k}+\beta_{k}\right)} .
$$

From (3.1) and (3.3), we get

$$
m_{h}\left(D_{n}, 0\right) \leq \frac{1}{4 \pi} \sum_{k=1}^{m} F\left(\alpha_{k}, \beta_{k}\right),
$$

where the function $F$ is defined by (1.12).

By Theorem [1.8 the function $F$ is concave for the considered values of $\alpha_{k}, \beta_{k}$. Therefore, since

$$
\sum_{k=1}^{m} \alpha_{k}=1, \quad \sum_{k=1}^{m} \beta_{k}=m / 2-1-2 A^{*} / \pi,
$$

inequality (1.13) implies

$$
\sum_{k=1}^{m} F\left(\alpha_{k}, \beta_{k}\right) \leq m F\left(1 / m, \beta_{0}\right)
$$

with the sign of equality if and only if

$$
\alpha_{1}=\ldots=\alpha_{m}=1 / m, \quad \beta_{1}=\ldots=\beta_{m}=\beta_{0},
$$

where

$$
\beta_{0}=1 / 2-1 / m-2 A^{*} / \pi m \text {. }
$$

Now (3.2), (3.4), and (3.5) combined with equality (2.20) and the monotonicity assertion of Lemma 2.3 lead to the following chain of inequalities:

$$
m_{h}\left(D_{n}, 0\right) \leq m_{h}\left(D_{m}\left(A^{*}\right), 0\right) \leq m_{h}\left(D_{n}\left(A^{*}\right), 0\right) \leq m_{h}\left(D_{n}(A), 0\right),
$$

which proves inequality (1.4).

To prove the uniqueness assertion of Theorem 1.1, assume that for $D_{n}$ considered in the proof above, (1.4) holds with the sign of equality. Then we must have the sign of equality in all the inequalities (3.1)-(3.6).

In particular, the second inequality in (3.6) becomes the equality $m_{h}\left(D_{m}\left(A^{*}\right), 0\right)$ $=m_{h}\left(D_{n}\left(A^{*}\right), 0\right)$, which by Lemma 2.3 implies that $m=n$. The latter easily implies that $D_{n}^{*}=D_{n}$. In particular, $A^{*}=A$.

Since $F$ is strictly concave and (3.4) holds with the sign of equality, we must have

$$
\alpha_{k}=1 / n, \quad \beta_{k}=1 / 2-1 / n-2 A / \pi n .
$$

Finally, since for all $k=1, \ldots, n,(3.3)$ holds with the sign of equality, it follows from Lemma 2.2 that for all $k=1, \ldots, n, T_{k}$ is an isosceles hyperbolic triangle having the angle $2 \pi / n$ at the origin and $h$-area $A / n$. This shows that $D_{n}$ is a regular hyperbolic $n$-gon centered at the origin. The proof of Theorem 1.1 is complete. 
Proof of Theorem 1.2. Since $R\left(D_{n}\right)$ is invariant under the isometries of the Euclidean plane, we may assume that $R\left(D_{n}\right)=\max _{z \in D_{n}} R\left(D_{n}, z\right)=R\left(D_{n}, 0\right)$. For $\rho>0$, let $D_{n, \rho}=\left\{z: \rho z \in D_{n}\right\}$. Then,

$$
\text { Area }\left(D_{n, \rho}\right)=\rho^{-2} \text { Area }\left(D_{n}\right), \quad R\left(D_{n, \rho}, 0\right)=\rho^{-1} R\left(D_{n}, 0\right) .
$$

If $\rho>0$ is large enough, then $\bar{D}_{n, \rho} \subset \mathbb{U}$. Let $a_{k}, k=1, \ldots, n$, denote the vertices of $D_{n}$ enumerated in the positive direction on $\partial D_{n}$. Let $D_{n, \rho}^{h}$ be a hyperbolic $n$-gon with vertices at the points $a_{k, \rho}=\rho^{-1} a_{k}$ enumerated in the positive direction on $\partial D_{n, \rho}^{h}$ and let $\widetilde{D}_{n, \rho}=\left\{z: \rho^{-1} z \in D_{n, \rho}^{h}\right\}$. One can show that for every sequence $\rho_{m} \rightarrow \infty$ the corresponding sequence of the family of the circular polygons $\widetilde{D}_{n, \rho_{m}}$ converges to the polygon $D_{n}$ in the sense that

$$
\max _{\zeta \in \partial \widetilde{D}_{n, \rho_{m}}} \min _{\omega \in \partial D_{n}}|\zeta-\omega| \rightarrow 0, \quad \max _{\zeta \in \partial D_{n}} \min _{\omega \in \partial \widetilde{D}_{n, \rho_{m}}}|\zeta-\omega| \rightarrow 0
$$

as $m \rightarrow \infty$. In particular, the sequence of domains $\widetilde{D}_{n, \rho_{m}}$ converges to the kernel $D_{n}$ in the Caratheodory sense, which implies the second limit relation in (3.7). Also, for all $\rho_{0}>0$ sufficiently large, there is $\varepsilon=\varepsilon\left(\rho_{0}\right)>0$ such that

$$
\left(1-\varepsilon\left(\rho_{0}\right)\right) \operatorname{Area}\left(D_{n, \rho}\right) \leq h \text {-area }\left(D_{n, \rho}^{h}\right) \leq\left(1+\varepsilon\left(\rho_{0}\right)\right) \operatorname{Area}\left(D_{n, \rho}\right)
$$

for all $\rho \geq \rho_{0}$, and such that $\lim _{\rho_{0} \rightarrow \infty} \varepsilon\left(\rho_{0}\right)=0$. Since $\rho^{2} \operatorname{Area}\left(D_{n, \rho}\right)=\operatorname{Area}\left(D_{n}\right)$, the latter inequalities imply

$$
\begin{aligned}
\left(1-\varepsilon\left(\rho_{0}\right)\right) \operatorname{Area}\left(D_{n}\right) & \leq{\varliminf_{\rho \rightarrow \infty} \rho^{2} h \text {-area }\left(D_{n, \rho}^{h}\right)} \leq \varlimsup_{\rho \rightarrow \infty} \rho^{2} h \text {-area }\left(D_{n, \rho}^{h}\right) \leq\left(1-\varepsilon\left(\rho_{0}\right)\right) \operatorname{Area}\left(D_{n}\right) .
\end{aligned}
$$

Letting $\rho_{0} \rightarrow \infty$, we get

$$
\lim _{\rho \rightarrow \infty} \rho^{2} h \text {-area }\left(D_{n, \rho}^{h}\right)=\operatorname{Area}\left(D_{n}\right)=A, \quad \lim _{\rho \rightarrow \infty} \rho R\left(D_{n, \rho}^{h}, 0\right)=R\left(D_{n}, 0\right) .
$$

Applying (1.4) with

$$
\beta=1 / 2-1 / n-q_{\rho}, \quad \text { where } q_{\rho}=2\left[h \text {-area }\left(D_{n, \rho}^{h}\right) /(\pi n)\right],
$$

for the polygon $D_{n, \rho}^{h}$, we obtain

$$
\rho^{2} R^{2}\left(D_{n, \rho}^{h}, 0\right) \leq \rho^{2} q_{\rho} \frac{\Gamma^{2}\left(1-\frac{1}{n}\right) \Gamma\left(1-q_{\rho}\right) \Gamma\left(\frac{2}{n}+q_{\rho}\right)}{\Gamma^{2}\left(1+\frac{1}{n}\right) \Gamma\left(1-\frac{2}{n}-q_{\rho}\right) \Gamma\left(1+q_{\rho}\right)} .
$$

Taking the limit in (3.8) as $\rho \rightarrow \infty$ and taking into account (3.7), we get the inequality

$$
R^{2}\left(D_{n}, 0\right) \leq \frac{2 A}{\pi n} \frac{\Gamma^{2}\left(1-\frac{1}{n}\right) \Gamma\left(\frac{2}{n}\right)}{\Gamma^{2}\left(1+\frac{1}{n}\right) \Gamma\left(1-\frac{2}{n}\right)} .
$$

Using Legendre's duplication formula $\Gamma(2 z)=2^{2 z-1} \pi^{-1 / 2} \Gamma(z) \Gamma(z+1 / 2)$, one can easily show that (3.9) is equivalent to (1.5)).

Since we used a passage to a limit to get (3.9), we lost the assertion on the cases of equality. To save this assertion, one can repeat some steps in the proof of Theorem 1.1. Namely, we can decompose the star of $D_{n}$ into $m$ trilaterals, write inequalities similar to (3.1), (3.4), (3.6), and then analyse the cases of equality in each of these inequalities. We are not going into detail since this analysis was already done in 31. 
Now we turn to the proof of Corollaries 1.1 and 1.2. The first inequality in (1.7) is an immediate consequence of Theorem 1.1. To prove the second one, we note that

$$
\frac{R_{h}^{2}\left(D_{n}(A)\right)}{A}=\frac{2}{\pi n} \frac{\Gamma^{2}\left(1-\frac{1}{n}\right) \Gamma\left(\frac{1}{2}+\frac{1}{n}+\beta\right) \Gamma\left(\frac{1}{2}+\frac{1}{n}-\beta\right)}{\Gamma^{2}\left(1+\frac{1}{n}\right) \Gamma\left(\frac{1}{2}-\frac{1}{n}+\beta\right) \Gamma\left(\frac{3}{2}-\frac{1}{n}-\beta\right)},
$$

where $\beta=1 / 2-1 / n-2 A / \pi n$. Logarithmic differentiation of (3.10) with respect to $\beta$ gives

$$
\frac{d}{d \beta} \log \frac{R_{h}^{2}\left(D_{n}(A)\right)}{A}=\psi\left(\frac{1}{2}+\frac{1}{n}+\beta\right)-\psi\left(\frac{1}{2}+\frac{1}{n}-\beta\right)-\psi\left(\frac{1}{2}-\frac{1}{n}+\beta\right)+\psi\left(\frac{3}{2}-\frac{1}{n}-\beta\right)>0
$$

since the function $\psi$ is increasing for the considered values of the parameters. This shows that the ratio $R_{h}^{2}\left(D_{n}(A)\right) / A$ strictly decreases for $A>0$, which in the limit as $A \rightarrow 0^{+}$gives the second inequality in (1.7).

The second and third inequalities in (1.8) are elementary. The proof of the first one is standard. We approximate $D$ with a sequence of hyperbolic polygons $D^{n}$ such that $D^{n} \subset D^{n+1} \subset D$ for $n=3,4, \ldots$, where $D^{n}$ has $n$ sides and $\bigcup_{n=3}^{\infty} D^{n}=D$. Let $s_{n}=h$-area $\left(D^{n}\right)$. Then $s_{n} \rightarrow h$-area $(D)$ and, it follows from the Caratheodory convergence theorem (see [16] Ch. 2.5]) that $R_{h}\left(D^{n}, z_{0}\right) \rightarrow R_{h}\left(D, z_{0}\right)$ as $n \rightarrow \infty$. Therefore,

$$
\frac{R_{h}^{2}\left(D, z_{0}\right)}{h \text {-area }(D)}=\lim _{n \rightarrow \infty} \frac{R_{h}^{2}\left(D^{n}, z_{0}\right)}{s_{n}} \leq \lim _{n \rightarrow \infty} \frac{R_{h}^{2}\left(D_{n}\left(s_{n}\right), z_{0}\right)}{s_{n}} .
$$

Using (3.10) one can find that the second limit in (3.11) is equal to $\left(\pi \cosh ^{2} \rho\right)^{-1}$.

\section{Dissymmetrization And Proof of Theorems [1.6 And 1.7}

A geometric transformation called dissymmetrization was introduced by V. N. Dubinin [10]. It uses the "cutting-gluing" technique to construct objects, like sets, functions, etc., from another set of objects of the same nature but having a rich group of symmetry. Below we define this transformation following the presentation in [11] and [32].

For a fixed positive integer $n$, let $\mathcal{G}_{n}$ be the group of isometries of $\mathbb{C}$ generated by reflections with respect to the lines $l_{k}=\{z: \Im(z \exp (\pi i(k-1) / n))=0\}$, $k=1, \ldots, n$. By a symmetrical structure $\left\{P_{k}\right\}_{k=1}^{N}$ we mean a collection of closed angles $P_{k}=\left\{z: \theta_{k}^{\prime} \leq \arg z \leq \theta_{k}^{\prime \prime}\right\}, \theta_{k}^{\prime}<\theta_{k}^{\prime \prime}$, satisfying the following conditions:

a) $\bigcup_{k=1}^{N} P_{k}=\mathbb{C}$

b) Interior $\left(P_{k} \cap P_{l}\right)=\emptyset \quad$ if $\quad k \neq l$,

c) $\left\{\varphi\left(P_{k}\right)\right\}_{k=1}^{N}=\left\{P_{k}\right\}_{k=1}^{N} \quad$ for any isometry $\varphi \in \mathcal{G}_{n}$.

A collection of mappings $\left\{\lambda_{k}\right\}_{k=1}^{N}$ of the form $\lambda_{k}(z)=z \exp \left(i \theta_{k}\right), \theta_{k} \in \mathbb{R}$, $k=1, \ldots, N$, is called dissymmetrization of the structure $\left\{P_{k}\right\}_{k=1}^{N}$ if the following conditions are fullfiled:

1) $\bigcup_{k=1}^{N} \lambda_{k}\left(P_{k}\right)=\mathbb{C}$,

2) for any non-empty intersection $S_{k, l}=\lambda_{k}\left(P_{k}\right) \cap \lambda_{l}\left(P_{l}\right), k, l=1, \ldots, N, k \neq l$, there exists an isometry $\varphi \in \mathcal{G}_{n}$ such that

$$
\varphi\left(\lambda_{k}^{-1}\left(S_{k, l}\right)\right)=\lambda_{l}^{-1}\left(S_{k, l}\right) .
$$


Let $E$ be a set on $\mathbb{C}$ invariant under the isometries of the group $\mathcal{G}_{n}$. If a symmetrical structure $\left\{P_{k}\right\}_{k=1}^{N}$ and its dissymmetrization $\left\{\lambda_{k}\right\}_{k=1}^{N}$ are defined, then dissymmetrization of $E$ is defined by

$$
\operatorname{Dis}(E)=\bigcup_{k=1}^{N} \lambda_{k}\left(E \cap P_{k}\right) .
$$

Note that Dis $(E)$ is open if $E$ is, but it might be disconnected containing multiply connected components even if $E$ is simply connected. The following lemma in an equivalent form for the conformal radius is proved by V. N. Dubinin.

Lemma 4.1 ([10, 11]). Let $D, 0 \in D \subset \mathbb{U}$, be a simply connected domain invariant under the isometries of the group $\mathcal{G}_{n}$. If $\mathrm{Dis}(D)$ is simply connected, then

$$
m_{h}(D, 0) \leq m_{h}(\operatorname{Dis}(D), 0)
$$

with the sign of equality if and only if $\mathrm{Dis}(D)$ coincides with $D$ up to rotation about the origin.

In contrast to symmetrization, application of dissymmetrization is not straightforward. Namely, for a given object and a relevant symmetric object, the characteristics of which we want to compare, we first need to prove the existence of a dissymmetrization transforming the symmetric object into the given one. To prove Theorem 1.6. we need Lemma 4.2 below, the proof of which is identical with the existence part of the proof of Theorem 1 in 32 if we replace in that theorem the Euclidean polygons and triangles with the hyperbolic ones.

Lemma 4.2 (cf. 32] Theorem 1]). For $0<\rho<\rho_{n}$, let $D_{n, \rho}$ be the regular hyperbolic n-gon circumscribed about the circle $C_{\rho}^{h}=C_{\rho}^{h}(0)$ such that $r=\tanh 2 \rho \in$ $\partial D_{n, \rho}$. For any hyperbolic n-gon $D_{n}$ circumscribed about $C_{\rho}^{h}$ there exists a symmetrical structure and its dissymmetrization such that $\operatorname{Dis}\left(D_{n, \rho}\right) \subset D_{n}$ with the strict inclusion except in the case when $D_{n}$ coincides with $D_{n, \rho}$ up to rotation about the origin.

Proof of Theorem 1.6. The proof follows directly from Lemmas4.1 and 4.2 Indeed, by Lemma 4.2 for a given $D_{n}$ satisfying the assumptions of the theorem, there is a dissymetrization of $D_{n, \rho}$ such that Dis $\left(D_{n, \rho}\right) \subset D_{n}$. Since the reduced $h$-module is a strictly increasing function of a domain,

$$
m_{h}\left(D_{n}, 0\right)>m_{h}\left(\operatorname{Dis}\left(D_{n, \rho}\right), 0\right)
$$

if $D_{n}$ is not a rotation of $D_{n, \rho}$. Inequality (4.2) combined with the inequality $m\left(\operatorname{Dis}\left(D_{n, \rho}\right), 0\right) \geq m\left(D_{n, \rho}, 0\right)$, which follows from Lemma 4.1 and relation (2.2) leads to (1.10) after an easy computation of $\beta$.

Dissymmetrization can be used whenever the relevant group of isometries is not trivial. We demonstrate its action on an elementary extremal problem on the reduced module of a trilateral needed for the proof of Theorem [1.7. This may help the reader get a better "feel" for what lies behind the somewhat complicated notations.

We fix $\alpha, 0<\alpha<1 / 2, r>0$, and $R>0$ such that $(r+R) \sin \alpha \pi<R$. Then for $\theta$ such that $0 \leq \theta \leq \alpha$ and $(r+R) \sin (\alpha+\theta) \pi \leq R$, there exists a circular triangle $T(\theta)$, lying on the Euclidean plane, with vertices $e_{1}=0, e_{2}=e_{2}(\theta), e_{3}=e_{3}(\theta)$, that has two sides $\overbrace{1} e_{2}$ and $\overbrace{1} e_{3}$ lying on the rays $l_{-\alpha}$ and $l_{\alpha}$, respectively, where 


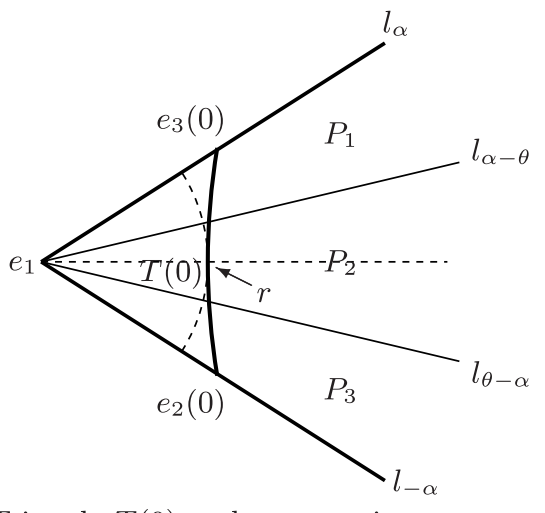

a) Triangle $T(0)$ and symmetric structure on $P_{\alpha}$

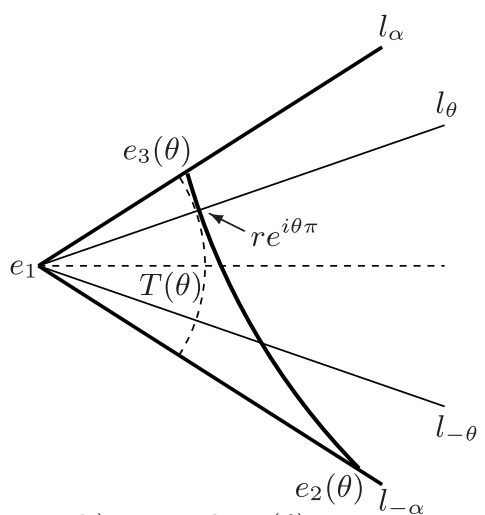

b) Triangle $T(\theta)$

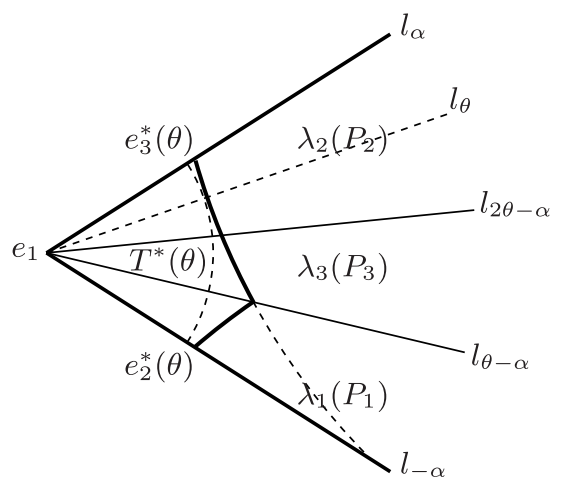

c) Dissymetrization of $T(0)$

FiguRE 5. Dissymmetrization

$l_{\gamma}=\{z: \arg z=\gamma \pi\}$, and whose third side $\widehat{e}_{2} e_{3}$ is a circular arc of radius $R$ that touches the circle $C_{r}=\{z:|z|=r\}$ from the outside at the point $r e^{i \theta \pi}$. See Figure 5, which also explains some notations used in the proofs of this section.

Lemma 4.3. Let $m(T(\theta))$ denote the reduced module of $T(\theta)$ at the vertex $e_{1}$. Then for $0<\theta \leq \alpha$,

$$
m(T(\theta))>m(T(0)) .
$$

Proof. Consider the angle $P_{\alpha}=\{z:|\arg z| \leq \alpha \pi\}$ with its group of isometries $\left\{\varphi_{0}, \varphi_{1}\right\}$ consisting of the identity mapping $\varphi_{0}$ and the mapping $\varphi_{1}$ that is the reflection with respect to the real axis. Choose a symmetrical structure on $P_{\alpha}$ consisting of three angles: $P_{1}=\{z:(\alpha-\theta) \pi \leq \arg z \leq \alpha \pi\}, P_{2}=\{z:|\arg z| \leq$ $(\alpha-\theta) \pi\}$, and $P_{3}=\{z:-\alpha \pi \leq \arg z \leq-(\alpha-\theta) \pi\}$. The collection of three rotations

$$
\lambda_{1}(z)=z \exp (-i(2 \alpha-\theta) \pi), \quad \lambda_{2}(z)=z \exp (i \theta \pi), \quad \lambda_{3}(z)=z \exp (i \theta \pi)
$$

defines the dissymmetrization of the structure $\left\{P_{1}, P_{2}, P_{3}\right\}$. Let $T^{*}(\theta)$ be the corresponding dissymmetrization of $T(0)$ considered as a trilateral with the vertex $e_{1}$ at $z=0$ and the opposite side $\left\{z \in \partial T^{*}(\theta):|\arg z|<\alpha \pi\right\}$. Let $e_{2}^{*}(\theta)$ and $e_{3}^{*}(\theta)$ denote the vertices of $T^{*}(\theta)$ that are different from $e_{1}$, and let $P_{k}^{*}=\lambda_{k}\left(P_{k}\right)$, 
$k=1,2,3$. Figure 55: shows how this dissymmetrization affects the considered triangles. The proof of (4.1) given in [10, 11, which also works for the reduced modules of trilaterals, shows that $m(T(0)) \leq m\left(T^{*}(\theta)\right)$. Geometrically it is clear that $T^{*}(\theta) \subset T(\theta)$ and this inclusion is strict. Therefore, $m\left(T^{*}(\theta)\right)<m(T(\theta))$ since the reduced module is a strictly monotonic function of a domain; see [35. Lemma 1.1]. This, combined with the previous inequality, proves the lemma.

Proof of Theorem 1.7. Let $D$ be a hyperbolic polygon with $m$ sides satisfying the assumptions of the theorem. If $m \leq n$, then it follows from Theorem 1.1 and Lemma 2.3 that

$$
m_{h}(D, 0) \leq m_{h}\left(D_{m}\left(\sigma_{m}\right), 0\right) \leq m_{h}\left(D_{n}\left(\sigma_{n}\right), 0\right),
$$

where $\sigma_{k}=\pi(k-2) / 4$ is the maximal hyperbolic area of a hyperbolic $k$-gon. Equality occurs in the first case if and only if $D$ is a rotation of $D_{m}\left(\sigma_{m}\right)$ around the origin and in the second case if and only if $m=n$. This, combined with equalities (2.2) and (2.20), proves the theorem in the case under consideration.

Let $m>n$. If $\rho<\rho_{n}$, then increasing the radius of the inscribed circle if necessary, we can easily find a new $m$-gon $D_{m}$ circumscribed about $C_{\rho_{n}}^{h}$ such that $D \subset D_{m}$ with the sign of strict inclusion. Since the reduced $h$-module is a strictly monotonic function of a domain,

$$
m_{h}(D, 0)<m_{h}\left(D_{m}, 0\right),
$$

and therefore to prove the theorem for $m>n$ we can restrict ourselves to polygons circumscribed about $C_{\rho_{n}}^{h}$.

The rectilinear segments joining $z=0$ with the vertices of $D_{m}$ and the segments joining $z=0$ with the points of the intersection $\partial D_{m} \cap C_{\rho_{n}}^{h}$ split $D_{m}$ into $2 m$ hyperbolic right triangles $T_{k}, k=1, \ldots, 2 m$, enumerated such that for every $k, 1 \leq$ $k \leq m, T_{k}$ and $T_{m+k}$ have a common hypotenuse. Then $T_{k}$ and $T_{m+k}$ are symmetric to each other with respect to their hypotenuse and therefore $m_{h}\left(T_{k} ; 0 \mid e_{2}^{k}, e_{3}^{k}\right)=$ $m_{h}\left(T_{m+k} ; 0 \mid e_{2}^{m+k}, e_{3}^{m+k}\right)$ for $k=1, \ldots, m$. Let $\alpha_{k} \pi$ denote the angle of $T_{k}$ at the vertex $z=0$. Since $D_{m}$ is circumscribed about $C_{\rho_{n}}^{h}$, it is easy to see that $0<\alpha_{k} \leq 1 / n$ and $\sum_{k=1}^{2 m} \alpha_{k}=2$. The system $T_{k}, k=1, \ldots, 2 m$, is a competing system of trilaterals for the domain $D_{m}$ in the sense of Theorem 2.1$]$ From this theorem using formula (2.18) for the reduced $h$-module of a hyperbolic right triangle we obtain

$$
m_{h}\left(D_{m}, 0\right) \leq(1 / 4) \sum_{k=1}^{2 m} \alpha_{k}^{2} m_{h}\left(T_{k} ; 0 \mid e_{2}^{k}, e_{3}^{k}\right)=(1 / 2 \pi) \sum_{k=1}^{m} F\left(\alpha_{k}, \beta_{k}\right),
$$

where $F$ is defined by (1.12) and $\beta_{k}=\beta\left(\alpha_{k}, \rho\right)$, where

$$
\beta(\alpha, \rho)=(1 / \pi) \cos ^{-1}(\sin \alpha \pi \cosh 2 \rho) .
$$

We claim that for a fixed $\rho>0$, the function $g(\alpha)=F(\alpha, \beta(\alpha, \rho))$ is strictly convex for $-1 / 2<\alpha<1 / 2$. Let $\alpha_{1}>\alpha_{2}>0,2 \alpha=\alpha_{1}+\alpha_{2}<1$, and let $\theta=\left(\alpha_{1}-\alpha_{2}\right) / 2$. Let $T(\theta)$ be the triangle defined in Lemma 4.3 The ray $l_{\theta}$ splits $T(\theta)$ into two triangles $T^{k}(\theta), k=1,2$, such that $T^{k}(\theta)$ has the angle $\alpha_{k} \pi$ at its vertex $z=0$. Let $T^{k}(0), k=1,2$, be similar triangles corresponding to the isosceles triangle $T(0)$ and let $m_{h}(T(\theta))$ and $m_{h}\left(T^{k}(\theta)\right)$ denote the reduced $h$-module of the 
corresponding triangle at its vertex $z=0$. From Theorem 2.2 and inequality (4.3) of Lemma 4.3, we get

$$
\begin{aligned}
(1 / 2) \alpha^{2} m_{h}\left(T^{1}(0)\right) & =\alpha^{2} m_{h}(T(0))<\alpha^{2} m_{h}(T(\theta)) \\
& \leq \alpha^{2}\left[\left(\alpha_{1} / 2 \alpha\right)^{2} m_{h}\left(T^{1}(\theta)\right)+\left(\alpha_{2} / 2 \alpha\right)^{2} m_{h}\left(T^{2}(\theta)\right)\right] \\
& =(1 / 4)\left(\alpha_{1}^{2} m_{h}\left(T^{1}(\theta)\right)+\alpha_{2}^{2} m_{h}\left(T^{2}(\theta)\right)\right),
\end{aligned}
$$

where the first equality follows from the assertion on the cases of equality of Theorem 2.2. the second relation follows from Lemma 4.3. and the third one follows again from Theorem 2.2 It follows from 2.18) that $\alpha^{2} m_{h}(T(0))=g(\alpha)$, $\alpha_{k}^{2} m_{h}\left(T^{k}(\theta)\right)=g\left(\alpha_{k}\right)$. Therefore (4.6) proves the convexity of $g(\alpha)$.

Now we return to the proof of the theorem. Since $0<\alpha_{k} \leq 1 / n$ and $m>n$, the row of $m$ elements $\left(\alpha_{1}, \ldots, \alpha_{m}\right)$ is majorized by the row $(1 / n, \ldots, 1 / n, 0, \ldots, 0)$ consisting of $n$ elements equal to $1 / n$ and $m-n$ zero elements. For the definition and properties of majorization we refer to [19, 26]. Since $g(\alpha)$ is convex, it follows from the well-known majorization theorem of Hardy, Littlewood, and Pólya, [19. Theorem 108, p. 89], that

$$
\sum_{k=1}^{m} F\left(\alpha_{k}, \beta_{k}\right)=\sum_{k=1}^{m} g\left(\alpha_{k}\right) \leq n g(1 / n)=n F(1 / n, \beta(1 / n))
$$

with the sign of equality if and only if $n$ of the values $\alpha_{k}$ are equal to $1 / n$ and the others are zero.

Equations (4.4)-(4.7) combined with equalities (2.2) and (2.20) lead to (1.11) with the sign of equality only for the regular $n$-gons.

One can compare the proof of the convexity property of function $g$ given above, which is purely geometric, with the highly analytic proof of necessary monotonicity and convexity results presented in Sections 5 and 6. An advantage of the geometric proof is that it does not require an explicit expression of the reduced modules and therefore can be applied to a wider variety of configurations when such an expression is not known.

\section{Absolutely monotonic functions}

The functions relevant to the conformal invariants usually have an amazingly regular behavior. Here and in Section 6, we show that the reduced modules of polygons not only satisfy the desired monotonicity and concavity conditions but are also absolutely monotonic. We recall that a function $f$ is said to be absolutely monotonic (increasing) on an interval $I=\{x: 0<x<c\}, c>0$, if $f$ has derivatives of all orders on $I$ that are all positive. Equivalently, $f$ is absolutely monotonic on $I$ if it can be represented there by a power series $f(x)=a_{0}+a_{1} x+$ $a_{2} x^{2}+\ldots$ with non-negative coefficients $a_{k}$, infinitely many of which are positive; cf. [13, p. 223]. The following three lemmas deal with the function $S$, defined by (2.17).

Lemma 5.1. For fixed $a, b, c>0$ such that $a+b<1 / 2, S=S(a, b,-c, t)$ is absolutely monotonic for $0<t<1$.

Proof. We write $S=S(a, b,-c, t)$ as

$$
S=c \log \frac{\Gamma(1+a) \Gamma(1 / 2-a+b t)}{\Gamma(1-a) \Gamma(1 / 2+a+b t)}-c \log \frac{\Gamma(1-a) \Gamma(1 / 2+a-b t)}{\Gamma(1+a) \Gamma(1 / 2-a-b t)},
$$


then apply the integral representation 3.413 [17, p.327],

$$
\log \frac{\Gamma(\mu) \Gamma(\beta+\gamma+\mu)}{\Gamma(\mu+\beta) \Gamma(\mu+\gamma)}=\int_{0}^{\infty} \frac{\left(1-e^{-\beta \tau}\right)\left(1-e^{-\gamma \tau}\right) e^{-\mu \tau}}{\tau\left(1-e^{-\tau}\right)} d \tau
$$

with $\mu=1+a, \beta=-2 a, \gamma=-1 / 2+b t$ for the first logarithm in (5.1) and with $\mu=1-a, \beta=2 a, \gamma=-1 / 2-b t$ for the second one. Note that (5.2) holds if

$$
\Re \mu>0, \quad \Re(\mu+\beta)>0, \quad \Re(\mu+\gamma)>0, \quad \Re(\mu+\beta+\gamma)>0 .
$$

Since for $a, b>0, a+b<1 / 2,0<t<1$, conditions (5.3) are satisfied, equations (5.1) and (5.2) lead to

$$
\begin{aligned}
S= & c \int_{0}^{\infty} \frac{\left(1-e^{2 a \tau}\right)\left(1-e^{(1 / 2-b t) \tau}\right) e^{-(1+a) \tau}}{\tau\left(1-e^{-\tau}\right)} d \tau \\
& -c \int_{0}^{\infty} \frac{\left(1-e^{-2 a \tau}\right)\left(1-e^{(1 / 2+b t) \tau}\right) e^{-(1-a) \tau}}{\tau\left(1-e^{-\tau}\right)} d \tau,
\end{aligned}
$$

which after some algebra becomes

$$
S=4 c \int_{0}^{\infty} \frac{\sinh a \tau\left(e^{\tau / 2} \cosh b t \tau-1\right)}{\tau\left(e^{\tau}-1\right)} d \tau
$$

We shall use the well-known Taylor expansions for the hyperbolic functions:

$$
\cosh (b t \tau)=\sum_{k=0}^{\infty} \frac{(b t)^{2 k}}{(2 k) !} \tau^{2 k}, \quad \sinh (a \tau)=\sum_{k=0}^{\infty} \frac{a^{2 k+1}}{(2 k+1) !} \tau^{2 k+1}, \quad \tau \in \mathbb{R} .
$$

Using the first expansion in (5.5), we obtain from (5.4)

$$
S=2 c \int_{0}^{\infty}\left(\sum_{k=0}^{\infty} t^{2 k} g_{k}(a, b, \tau)\right) d \tau
$$

where

$$
g_{0}=\frac{2 \sinh a \tau\left(e^{\tau / 2}-1\right)}{\tau\left(e^{\tau}-1\right)}, \quad g_{k}=\frac{b^{2 k} \tau^{2 k-1} \sinh a \tau}{(2 k) ! \sinh (\tau / 2)}, \quad k=1,2, \ldots
$$

Note that for fixed $a, b$, and $t$ satisfying the assumptions of the lemma and for $0 \leq \tau<\infty$, the sum $\sum_{k=0}^{\infty} t^{2 k} g_{k}(a, b, \tau)$ converges to the integrand in (5.4), which is continuous and integrable in $0 \leq \tau<\infty$. Therefore, since, for fixed $a$ and $b$, and for $k=0,1 \ldots, g_{k}$ is a continuous non-negative function of $\tau$ for $0 \leq \tau<\infty$, the series in (5.6) can be integrated termwise; see, for instance, [20, p. 306]. The termwise integration gives a representation of $S$ as a power series in terms of $t$ :

$$
S=2 c \sum_{k=0}^{\infty} t^{2 k} \int_{0}^{\infty} g_{k}(a, b, \tau) d \tau
$$

with positive even coefficients and zero odd coefficients, which proves the desired absolute monotonicity of $S$.

Lemma 5.2. For a fixed $\omega>1$ and $c>0$, the function $S=S(a, 1 / 2-a \omega,-c, 1)$ is absolutely monotonic for $0<a<1 /(1+\omega)$.

Proof. Using the identity $z \Gamma(z)=\Gamma(1+z)$, we can represent $S(a, 1 / 2-a \omega,-c, 1)$ as

$$
S(a, 1 / 2-a \omega,-c, 1)=c S_{1}(a, \omega)+c \log ((\omega+1) /(\omega-1))
$$


where

$$
S_{1}=\log \frac{\Gamma(1+a) \Gamma(1-a+a \omega)}{\Gamma(1-a) \Gamma(1+a+a \omega)}-\log \frac{\Gamma(1-a) \Gamma(1+a-a \omega)}{\Gamma(1+a) \Gamma(1-a-a \omega)} .
$$

Applying (5.2) with $\mu=1+a, \beta=-2 a, \gamma=a \omega$ for the first logarithm in (5.7) and with $\mu=1-a, \beta=2 a, \gamma=-a \omega$ for the second one, we obtain after an obvious simplification

$$
S_{1}=4 \int_{0}^{\infty} \frac{\sinh a \tau(\cosh a \omega \tau-1)}{\tau\left(e^{\tau}-1\right)} d \tau .
$$

Using the Cauchy product of the Taylor expansions (5.5) with $b=a, t=\omega$, each of which has non-negative terms for the considered values of the parameters, we obtain from (5.8)

$$
S_{1}(a, \omega)=4 \int_{0}^{\infty}\left(\sum_{k=0}^{\infty} a^{2 k+3} p_{k}(\omega, \tau)\right) d \tau
$$

where

$$
p_{k}(\omega, \tau)=\frac{\tau^{2 k+2}}{e^{\tau}-1} \sum_{j=0}^{k} \frac{\omega^{2 k-2 j+2}}{(2 j+1) !(2 k-2 j+2) !}, \quad k=0,1,2, \ldots
$$

The infinite sum in (5.9) converges to the integrand in (5.8), which is continuous and integrable in $0 \leq \tau<\infty$. Therefore since for fixed $\omega$ and for $k=0,1,2, \ldots, p_{k}$ is a continuous non-negative function of $\tau$ for $0 \leq \tau<\infty$, the series in (5.9) can be integrated termwise, which gives a representation of $S_{1}$ as a power series in terms of $a$ :

$$
S_{1}=4 \sum_{k=0}^{\infty} a^{2 k+3} \int_{0}^{\infty} p_{k}(\omega, \tau) d \tau
$$

with positive odd coefficients (except for the first one) and zero even coefficients. This proves that $S_{1}$, and therefore $S$, is absolutely monotonic for $0<a<1 /(1+\omega)$.

Representation (5.2) can be used to prove the following partial result in the direction of Theorem 1.8, which shows that (1.13) holds for all $\left(\alpha_{k}, \beta_{k}\right) \in \Pi$ such that $\alpha_{1} \leq \alpha_{2} \leq \ldots \leq \alpha_{m}$ and $\beta_{1} \leq \beta_{2} \leq \ldots \leq \beta_{m}$.

Lemma 5.3. For fixed $\alpha>0, \beta \geq 0, \gamma \geq 0, \delta \geq 0$ such that $\beta+\delta>0, \alpha+\beta+\gamma+\delta<$ $1 / 2$, the function $S=S(\alpha+\beta x, \gamma+\delta x,-(\alpha+\beta x), 1)$ is absolutely monotonic on $0<x<1$.

Proof. Note that (5.4) holds even if $t=1$ or $b=0$. Applying (5.4) with $a=\alpha+\beta x$, $b=\gamma+\delta x, c=a$, and $t=1$, we get

$$
S=4 a \int_{0}^{\infty} \frac{\sinh a \tau\left(e^{\tau / 2} \cosh b \tau-1\right)}{\tau\left(e^{\tau}-1\right)} d \tau .
$$

Using the Cauchy product of the Taylor expansions 5.5 with $t=1$, we obtain from 5.10

$$
S=4 \int_{0}^{\infty}\left(\sum_{k=0}^{\infty}\left(r_{k}(\tau) \frac{a^{2 k+2}}{(2 k+1) !}+q_{k}(\tau) \sum_{j=0}^{k} \frac{a^{2 j+2} b^{2(k-j+1)}}{(2 j+1) !(2(k-j+1)) !}\right)\right) d \tau
$$


where

$$
r_{k}(\tau)=\frac{\left(e^{\tau / 2}-1\right) \tau^{2 k}}{e^{\tau}-1}, \quad q_{k}(\tau)=\frac{e^{\tau / 2} \tau^{2 k+2}}{e^{\tau}-1}, \quad k=0,1,2, \ldots
$$

The infinite sum in (5.11) converges to the integrand in (5.10), which is continuous and integrable in $0 \leq \tau<\infty$. Since all the terms in this sum are continuous non-negative functions, the sum of which is integrable in $0 \leq \tau<\infty$ for the considered values of the parameters, it follows that (5.11) can be integrated termwise. This leads to the representation of $S$ as a power series in variables $a$ and $b$ :

$$
S=4 \sum_{k=0}^{\infty}\left(\frac{R_{k}}{(2 k+1) !} a^{2 k+2}+Q_{k} \sum_{j=0}^{k} \frac{a^{2 j+2} b^{2(k-j+1)}}{(2 j+1) !(2(j-k+1)) !}\right),
$$

with

$$
R_{k}=\int_{0}^{\infty} \frac{\left(e^{\tau / 2}-1\right) \tau^{2 k}}{e^{\tau}-1} d \tau, \quad Q_{k}=\int_{0}^{\infty} \frac{\tau^{2 k+2} e^{\tau / 2}}{e^{\tau}-1} d \tau, \quad k=0,1,2, \ldots,
$$

which converges for the considered values of the parameters.

Since $a^{n}=(\alpha+\beta x)^{n}, b^{m}=(\gamma+\delta x)^{m}$ and since $\alpha, \beta, \gamma$, and $\delta$ all are nonnegative, it follows from (5.12) and the binomial theorem that

$$
S=\sum_{k=0}^{\infty} m_{k}(\alpha, \beta, \gamma, \delta) x^{k}
$$

where $m_{k}(\alpha, \beta, \gamma, \delta) \geq 0$ for all $k \geq 0$ and all considered values of $\alpha, \beta, \gamma$, and $\delta$. In addition, since at least one of the parameters $\beta$ and $\delta$ is positive, it follows that $m_{k_{s}}>0$ for some subsequence $k_{s}$ of indices. Note also that the infinite sum in (5.13) converges for all $x \in(0,1)$. Since all the coefficients $m_{k}$ in (5.13) are non-negative and since there is a subsequence of positive coefficients, the desired absolute monotonicity of $S$ follows.

\section{Proof of Theorem 1.8}

To prove the concavity property of $F$, defined by equation (1.12), we apply the standard second derivative test. Let $f_{0}=F_{\alpha, \alpha}^{\prime \prime}, f_{1}=F_{\alpha, \beta}^{\prime \prime}, f_{2}=F_{\beta, \beta}^{\prime \prime}$ denote the corresponding second derivatives. Then after straightforward differentiation, we find

$$
\begin{aligned}
f_{0}= & -4 \psi(1-\alpha)-4 \psi(1+\alpha)+2 \alpha \psi^{\prime}(1-\alpha)-2 \alpha \psi^{\prime}(1+\alpha) \\
& +2\left(\psi\left(\frac{1}{2}+\alpha+\beta\right)+\psi\left(\frac{1}{2}+\alpha-\beta\right)+\psi\left(\frac{1}{2}-\alpha-\beta\right)+\psi\left(\frac{1}{2}-\alpha+\beta\right)\right) \\
& +\alpha\left(\psi^{\prime}\left(\frac{1}{2}+\alpha+\beta\right)+\psi^{\prime}\left(\frac{1}{2}+\alpha-\beta\right)-\psi^{\prime}\left(\frac{1}{2}-\alpha-\beta\right)-\psi^{\prime}\left(\frac{1}{2}-\alpha+\beta\right)\right), \\
f_{1}= & \psi\left(\frac{1}{2}+\alpha+\beta\right)-\psi\left(\frac{1}{2}+\alpha-\beta\right)+\psi\left(\frac{1}{2}-\alpha-\beta\right)-\psi\left(\frac{1}{2}-\alpha+\beta\right) \\
& +\alpha\left(\psi^{\prime}\left(\frac{1}{2}+\alpha+\beta\right)-\psi^{\prime}\left(\frac{1}{2}+\alpha-\beta\right)-\psi^{\prime}\left(\frac{1}{2}-\alpha-\beta\right)+\psi^{\prime}\left(\frac{1}{2}-\alpha+\beta\right)\right), \\
f_{2}= & \alpha\left(\psi^{\prime}\left(\frac{1}{2}+\alpha+\beta\right)+\psi^{\prime}\left(\frac{1}{2}+\alpha-\beta\right)-\psi^{\prime}\left(\frac{1}{2}-\alpha-\beta\right)-\psi^{\prime}\left(\frac{1}{2}-\alpha+\beta\right)\right) .
\end{aligned}
$$


We recall that $\alpha, \beta$ satisfy the inequalities

$$
\alpha>0, \quad \beta \geq 0, \quad \alpha+\beta<1 / 2 .
$$

Since $\psi^{\prime}(x)$ strictly decreases for $x>0$, it follows that $f_{2}<0$ for all $\alpha, \beta$ satisfying (6.1).

Let

$$
\Phi=\Phi(\alpha, \beta)=f_{2} f_{0}-f_{1}^{2} .
$$

Since $f_{2}<0$, to prove the concavity of $F$, we need to show that

$$
\Phi(\alpha, \beta)>0
$$

for all $\alpha$ and $\beta$ satisfying (6.1). Note that $\Phi$ is an even function in $\alpha$ and $\beta$ and

$$
\Phi(0, \beta)=0 \text { for } 0 \leq \beta<1 / 2 .
$$

Therefore (6.2) follows from the next much stronger result.

Theorem 6.1. For every fixed $\beta, 0 \leq \beta<1 / 2, \Phi(\alpha, \beta)$ is absolutely monotonic on $0<\alpha<1 / 2-\beta$.

Proof. For a fixed $\beta, 0 \leq \beta<1 / 2, \Phi(z, \beta)$ is analytic as a function of $z$ in the disk $\{z:|z|<1 / 2-\beta\}$. Since $\Phi$ is even, all its odd derivatives at $\alpha=0$ are zero:

$$
\frac{\partial^{2 j+1} \Phi(0, \beta)}{\partial \alpha^{2 j+1}}=0 \text { for } j=0,1,2, \ldots
$$

Therefore $\Phi$ can be represented as

$$
\Phi(\alpha, \beta)=\sum_{j=1}^{\infty} \frac{R_{j}(\beta)}{(2 j) !} \alpha^{2 j}, \quad 0 \leq \alpha<1 / 2-\beta .
$$

To prove the desired absolute monotonicity of $\Phi$, it is enough to show that

$$
R_{j}(\beta)>0 \quad \text { for } \quad j=1,2, \ldots \quad \text { and } \quad 0 \leq \beta<1 / 2 .
$$

To find the even derivatives $R_{j}(\beta)=\frac{\partial^{2 j} \Phi(0, \beta)}{\partial \alpha^{2 j}}, j=1,2, \ldots$, we use the Leibniz rule for the $n$-th derivative of the product of two functions. Then we obtain

$$
R_{j}(\beta)=16 L_{j}^{1}(u(\beta)),
$$

where

$$
u(\beta)=-\psi(1 / 2-\beta)-\psi(1 / 2+\beta)+2 \psi(1), \quad 0 \leq \beta<1 / 2,
$$

and the differential operator $L_{j}^{1}$ is defined by

$$
L_{j}^{1}=L_{j}^{0}+2 \sum_{k=0}^{j-1}\left(\begin{array}{c}
2 j \\
2 k
\end{array}\right)(j-k)(k+1) \nu_{k} \psi^{(2 k)}(1) D^{2 j-2 k}, \quad j=1,2, \ldots,
$$

where $D$ is the derivative operator, $\nu_{0}=0, \nu_{k}=1$ for $k=1,2, \ldots$ and

$$
\begin{aligned}
L_{j}^{0}= & \sum_{k=0}^{j-1}\left(\begin{array}{c}
2 j \\
2 k
\end{array}\right)(j-k)(k+1) D^{2 j-2 k} D^{2 k} \\
& -\sum_{k=0}^{j-1}\left(\begin{array}{c}
2 j \\
2 k+1
\end{array}\right)(j-k)(k+1) D^{2 j-2 k-1} D^{2 k+1} .
\end{aligned}
$$

To prove (6.3), we consider the function

$$
v(\beta)=-(\beta-1 / 2)^{-1} .
$$


It follows from Lemma 6.1 below that

$$
L_{j}^{0}(v(\beta))=0 \quad \text { for } \quad 0 \leq \beta<1 / 2 \quad \text { and } j=1,2, \ldots
$$

Hence, (6.3) is equivalent to the inequality

$$
L_{j}^{1}(u(\beta))>L_{j}^{0}(v(\beta)), \quad 0 \leq \beta<1 / 2 \quad \text { and } j=1,2, \ldots .
$$

In its turn, (6.6) follows from the inequalities (6.7) and (6.8) of Lemma 6.2 below, the proof of which finishes the proof of Theorem 6.1 and therefore the proof of Theorem 1.8.

Lemma 6.1. For all positive integers $j$,

$$
L_{j}^{0}(v(\beta))=0 \quad \text { on } \quad 0 \leq \beta<1 / 2,
$$

where $v$ is defined by (6.5).

Proof. Since $D^{n} v(\beta)=(-1)^{n+1} n !(\beta-1 / 2)^{-(n+1)}$, we have

$$
\begin{aligned}
& L_{j}^{0}(v(\beta))= \sum_{k=0}^{j-1}\left(\begin{array}{c}
2 j \\
2 k
\end{array}\right)(j-k)(k+1)(-1)^{2 j-2 k+1}(2 j-2 k) ! \\
& \quad \times(\beta-1 / 2)^{-(2 j-2 k+1)}(-1)^{2 k+1}(2 k) !(\beta-1 / 2)^{-(2 k+1)} \\
& \quad-\sum_{k=0}^{j-1}\left(\begin{array}{c}
2 j \\
2 k+1
\end{array}\right)(j-k)(k+1)(-1)^{2 j-2 k}(2 j-2 k-1) ! \\
& \quad \times(\beta-1 / 2)^{-(2 j-2 k)}(-1)^{2 k+2}(2 k+1) !(\beta-1 / 2)^{-(2 k+2)} \\
&=(2 j) !(\beta-1 / 2)^{-(2 j+2)} \sum_{k=0}^{j-1}[(j-k)(k+1)-(j-k)(k+1)]=0 .
\end{aligned}
$$

Lemma 6.2. For all $0 \leq \beta<1 / 2$ and all non-negative integers $m$,

$$
\begin{gathered}
D^{2 m+1} v(\beta)>D^{2 m+1} u(\beta) \geq 0, \\
D^{2 m} u(\beta) \geq D^{2 m} u(\beta)+2 \nu_{m} \psi^{(2 m)}(1)>D^{2 m} v(\beta)>0,
\end{gathered}
$$

where $u$ and $v$ are defined by (6.4) and (6.5), respectively, $\nu_{0}=0$, and $\nu_{k}=1$, $k=1,2, \ldots$.

Proof. Using the recurrence formula $\psi(z+1)=\psi(z)+z^{-1}$, [5, p. 16], we can write

$$
u(\beta)=-(\beta-1 / 2)^{-1}-\psi(3 / 2-\beta)-\psi(1 / 2+\beta)+2 \psi(1) .
$$

Since $v(\beta)=-(\beta-1 / 2)^{-1}$ and since the function $\psi(3 / 2-z)+\psi(1 / 2+z)$ is analytic in the disk $\{z:|z-1 / 2|<1 / 2\}$, we have

$$
\begin{aligned}
u(\beta) & =v(\beta)-\sum_{k=0}^{\infty} \frac{(-1)^{k} \psi^{(k)}(1)+\psi^{(k)}(1)}{k !}(\beta-1 / 2)^{k}+2 \psi(1) \\
& =v(\beta)-2 \sum_{k=1}^{\infty} \frac{\psi^{(2 k)}(1)}{(2 k) !}(\beta-1 / 2)^{2 k} .
\end{aligned}
$$

Since

$$
\psi^{(n)}(1)=(-1)^{n+1} n ! \zeta(n+1), \quad n=1,2, \ldots,
$$


([5, p. 45]) where $\zeta$ denotes the Riemann zeta function, (6.9) gives

$$
u(\beta)=v(\beta)+2 \sum_{k=1}^{\infty} \zeta(2 k+1)(\beta-1 / 2)^{2 k} .
$$

Note that for $k \geq 1, \zeta(2 k+1)>0$ and that the sum $\sum_{k=1}^{\infty} \zeta(2 k+1)(z-1 / 2)^{2 k}$ converges uniformly on any closed disk $\{z:|z-1 / 2| \leq \rho\}$ with $0<\rho<1 / 2$.

Differentiating (6.10) $2 m+1$ times with respect to $\beta$, we get for $0<\beta<1 / 2$,

$$
\begin{aligned}
D^{2 m+1} v(\beta) & >D^{2 m+1} v(\beta)+2 \sum_{k=m+1}^{\infty} \zeta(2 k+1) \frac{(2 k) !}{(2 k-2 m-1) !}(\beta-1 / 2)^{2 k-2 m-1} \\
& =D^{2 m+1} u(\beta)=\psi^{(2 m+1)}(1 / 2-\beta)-\psi^{(2 m+1)}(1 / 2+\beta) \geq 0,
\end{aligned}
$$

which proves (6.7). The latter inequality in this chain follows from the well-known representation for the polygamma function, [5, p. 45]:

$$
\psi^{(n)}(z)=(-1)^{n+1} n ! \sum_{k=0}^{\infty}(z+k)^{-(n+1)} .
$$

Now we turn to (6.8). The case $m=0$, when $\nu_{0}=0$, follows directly from (6.10). To prove (6.8) in the case $m=1,2, \ldots$, we differentiate (6.10) $2 m$ times. Then we get

$$
\begin{aligned}
0<- & (2 m) !(\beta-1 / 2)^{-(2 m+1)}=D^{2 m} v(\beta) \\
= & D^{2 m} u(\beta)-2 \sum_{k=m}^{\infty} \zeta(2 k+1) \frac{(2 k) !}{(2 k-2 m) !}(\beta-1 / 2)^{2 k-2 m} \\
= & D^{2 m} u(\beta)+2 \psi^{(2 m)}(1)+2(2 m) ! \zeta(2 m+1) \\
& -2 \sum_{k=m}^{\infty} \zeta(2 k+1) \frac{(2 k) !}{(2 k-2 m) !}(\beta-1 / 2)^{2 k-2 m} \\
= & D^{2 m} u(\beta)+2 \psi^{(2 m)}(1)-2 \sum_{k=m+1}^{\infty} \zeta(2 k+1) \frac{(2 k) !}{(2 k-2 m) !}(\beta-1 / 2)^{2 k-2 m} \\
< & D^{2 m} u(\beta)+2 \psi^{(2 m)}(1) .
\end{aligned}
$$

The lemma is proved.

\section{REFERENCES}

[1] L. V. Ahlfors, Conformal invariants: topics in geometric function theory. McGraw-Hill, New York, 1973. MR0357743 (50:10211)

[2] H. Alzer, On some inequalities for the gamma and psi functions. Math. Comp. 66 (1997), 373-389. MR1388887 (97e:33004)

[3] G. D. Anderson, M. K. Vamanamurthy, and M. K. Vuorinen, Conformal invariants, inequalities, and quasiconformal maps. John Wiley \& Sons, Inc., New York, 1997. MR1462077 (98h:30033)

[4] G. E. Andrews, R. Askey, and R. Roy, Special functions. Cambridge University Press, Cambridge, 1999. MF $1688958(2000 \mathrm{~g}: 33001)$

[5] H. Bateman and A. Erdelyi, Higher transcendental functions. Vol. 1. McGraw-Hill, New York, 1953. MR 0058756 (15:419i)

[6] A. F. Beardon, The geometry of discrete groups. Corrected reprint of the 1983 original. Graduate Texts in Mathematics, 91, Springer-Verlag, New York, 1995. MR.1393195|(97d:22011) 
[7] K. Bezdek, Ein elementarer Beweis für die isoperimetrische Ungleichung in der euklidischen und hyperbolischen Ebene. Ann. Univ. Sci. Budap. Rolando Eötvös, Sect. Math. 27 (1984), 107-112. MF 0823098 (87c:52016)

[8] R. Bonola, Non-Euclidean geometry, a critical and historical study of its developments. Dover Publications Inc., New York, 1955. MR0070197 (16:1145b)

[9] Yu. D. Burago and V. A. Zalgaller, Geometric inequalities. Grundlehren der Mathematischen Wissenschaften 285, Springer-Verlag, New York, 1988. MR0936419 (89b:52020)

[10] V. N. Dubinin, On the change in harmonic measure under symmetrization. Mat. Sb. 124 (166) (1984), 277-279; English transl. Math. USSR Sb. 52 (1985), 267-273. MR0746071 $(85 \mathrm{j}: 30049)$

[11] Symmetrization in geometric theory of functions of a complex variable. Uspekhi Mat. Nauk 49 (1994), 3-76; English transl. Russian Math. Surveys 49(1) (1994), 1-79. MR1307130 (96b:30054)

[12] P. L. Duren, Univalent functions. Springer-Verlag, New York, 1983. MR0708494 (85j:30034)

[13] W. Feller, An introduction to probability theory and its applications, Vol. 2, 2nd ed. John Willey \& Sons, Inc., 1971. MR0270403 (42:5292)

[14] L. R. Ford, Automorphic functions, 2nd ed. Chelsea, New York, 1951.

[15] A. E. Fryntov, A note on a harmonic measure estimate and a conjecture of J. Velling, preprint.

[16] G. M. Goluzin, Geometric theory of functions of a complex variable. Translations of Mathematical Monographs 26 AMS, Providence, R.I. (1969). MR0247039 (40:308)

[17] I. S. Gradshteyn and I. M. Ryzhik, Table of integrals, series, and products, Corrected and enlarged ed., Academic Press, New York, 1980. MF 0582453 (81g:33001)

[18] M. J. Greenberg, Euclidean and non-Euclidean geometries: Development and history, 3rd ed., W. H. Freeman and Co., New York, 1993. MR.1261866 (94k:51001)

[19] G. H. Hardy, J. E. Littlewood, and G. Pólya, Inequalities, 2nd ed. Cambridge Univ. Press, Cambridge, 1952. MR0046395 (13:727e)

[20] E. W. Hobson, The theory of functions of a real variable and the theory of Fourier series, Vol. 2, 3rd ed. Dover Publications, 1927. MR0092829 (19:1166b)

[21] J. A. Jenkins, Univalent functions and conformal mapping, 2nd ed. Springer, Berlin, 1965. MR0096806 (20:3288)

[22] W. Koppenfels and F. Stallmann, Praxis der konformen Abbildung. Springer-Verlag, Berlin, 1959. MR0107698 (21:6421)

[23] H.-T. Ku, M.-C. Ku, and X.-M. Zhang, Isoperimetric inequalities on surfaces of constant curvature. Canad. J. Math. 49 (1997), 1162-1187. MR.1611644 (99i:51020)

[24] R. Kühnau, Zum konformen Radius bei nullwinkligen Kreisbogendreiecken. Mitt. Math. Sem. Giessen 211 (1992), 19-24. MR1188834 (93k:30011)

[25] O. Lehto, Univalent functions and Teichmüller spaces. Springer-Verlag, New York, 1987. MR.0867407 (88f:30073)

[26] A.W. Marshall and I. Olkin, Inequalities: theory of majorization and its applications. Mathematics in Science and Engineering, 143, Academic Press, Inc. [Harcourt Brace Jovanovich, Publishers], New York-London, 1979. MR0552278 (81b:00002)

[27] J. Milnor, Hyperbolic geometry: The first 150 years. Bulletin (New Series) of AMS 6 (1982), 9-24. MR0634431 (82m:57005)

[28] G. Pólya and G. Szegö, Isoperimetric inequalities in mathematical physics. Princeton University Press, Princeton, 1951. MR 0043486 (13:270d)

[29] Chr. Pommerenke, Univalent functions. Vandenhoeck and Ruprecht, Göttingen, 1975. MR 0507768(58:22526)

[30] B. A. Rosenfeld, A history of non-Euclidean geometry. Springer-Verlag, New York, 1988. MR0959136 (89k:01001)

[31] A. Yu. Solynin, Solution of a Pólya-Szegö isoperimetric problem. Zap. Nauchn. Semin. LOMI 168 (1988), 140-153; English transl. J. Soviet Math. 53 (1991), 311-320. MR0982489 (90h:30059)

[32] - Isoperimetric inequalities for polygons and dissymmetrization. Algebra i Analiz 4 (1992), no. 2, 210-234; English transl. St. Petersburg Math. J. 4 (1993), no. 2, 377-396. MR.1182401 (93i:52014)

[33] , Some extremal problems for circular polygons. Zap. Nauchn. Semin. POMI, 206 (1993), 127-136; English transl. J. Math. Sci. 80 (1996), 1956-1961. MR 1255321 (94m:30048) 
[34] Some extremal problems on the hyperbolic polygons. Complex Variables Theory Appl. 36 (1998), 207-231. MR1671474 (99j:30030)

[35] _ Modules and extremal metric problems. Algebra i Analiz 11 (1999), no. 1, 3-86; English transl. St. Petersburg Math. J. 11 (2000), no. 1, 1-65. MR.1691080 (2001b:30058)

[36] A. Yu. Solynin and V. A. Zalgaller, An isoperimetric inequality for logarithmic capacity of polygons. Ann. of Math. 159 (2004), 277-303. MR2052355(2005a:31002)

[37] S. Stahl, The Poincaré half-plane: A gateway to modern geometry. Jones and Bartlett Publishers, Boston, 1993. MF,1217085 (94c:51041)

Department of Mathematics and Statistics, Texas Tech University, Box 41042, LubBOCK, TEXAS 79409

E-mail address: barnard@math.ttu.edu

Department of Mathematics and Statistics, Texas Tech University, Box 41042, LubBOCK, TEXAS 79409

E-mail address: phadjico@math.ttu.edu

Steklov Institute of Mathematics at St. Petersburg, Russian Academy of Sciences, Fontanka 27, St. Petersburg, 191011, Russia

Current address: Department of Mathematics and Statistics, Texas Tech University, Box 41042, Lubbock, Texas 79409

E-mail address: solynin@math.ttu.edu 